\title{
TORELLI THEOREM FOR GRAPHS AND TROPICAL CURVES
}

\author{
LUCIA CAPORASO AND FILIPPO VIVIANI
}

October 30, 2018

\begin{abstract}
Algebraic curves have a discrete analogue in finite graphs. Pursuing this analogy we prove a Torelli theorem for graphs. Namely, we show that two graphs have the same Albanese torus if and only if the graphs obtained from them by contracting all separating edges are 2-isomorphic. In particular, the strong Torelli theorem holds for 3-connected graphs. Next, using the correspondence between compact tropical curves and metric graphs, we prove a tropical Torelli theorem giving necessary and sufficient conditions for two tropical curves to have the same principally polarized tropical Jacobian. By contrast, we prove that, in a suitably defined sense, the tropical Torelli map has degree one. Finally we describe some natural posets associated to a graph and prove that they characterize its Delaunay decomposition.
\end{abstract}

\section{INTRODUCTION}

The analogy between graphs and algebraic curves has been a source of inspiration both in combinatorics and algebraic geometry. In this frame of mind, M. Kotani and T. Sunada (see [KS00]) introduced the Albanese torus, $\operatorname{Alb}(\Gamma)$, and the Jacobian torus, $\operatorname{Jac}(\Gamma)$, of a graph $\Gamma$; see section 2.1 for the precise definition.

By $\left[\mathrm{KS} 00, \operatorname{Alb}(\Gamma)\right.$ and $\operatorname{Jac}(\Gamma)$ are dual flat tori of dimension equal to $b_{1}(\Gamma)$, the first Betti number of $\Gamma$. As $b_{1}(\Gamma)$ is the maximum number of linearly independent cycles in $\Gamma$, it can be viewed as the analog for a graph of the genus of a Riemann surface. In analogy with the classical Torelli theorem for curves, it is natural to ask the following question:

Problem 1. When are two graphs $\Gamma$ and $\Gamma^{\prime}$ such that $\operatorname{Alb}(\Gamma) \cong \operatorname{Alb}\left(\Gamma^{\prime}\right)$ ?

There exist in the literature other versions of such a problem (see for example BdlHN97, or [BN07); the statement of Problem 1 is due to T. Sunada. One of the goals of this paper is to answer the above question. In our Theorem 3.1.1, we prove that $\operatorname{Alb}(\Gamma) \cong \operatorname{Alb}\left(\Gamma^{\prime}\right)$ if and only if the two graphs obtained from $\Gamma$ and $\Gamma^{\prime}$ by contracting all of their separating edges are cyclically equivalent (or 2-isomorphic, cf. Definition 2.2.3).

Using a result of Whitney, we obtain that the Torelli theorem is true for 3connected graphs; see Corollary 3.1.2 This answers a problem implicitly posed in [BdlHN97, Page 197], where the authors ask, albeit indirectly, whether there exist two non isomorphic, 3-connected graphs with isomorphic Albanese torus.

Let us now turn to another, recently discovered aspect of the analogy between graphs and curves, that is, the tight connection between tropical curves and graphs. By results of G. Mikhalkin and I. Zharkov, see [Mik06] and [MZ07, there exists a natural bijection between the set of tropical equivalence classes of compact tropical curves and metric graphs all of whose vertices have valence at least 3 .

Observe now that compact tropical curves, just like compact Riemann surfaces, are endowed with a Jacobian variety, which is a principally polarized tropical Abelian variety; see Section 4 for details. The following Torelli-type question arises 
Problem 2. Can two compact tropical curves have isomorphic Jacobian varieties? If so, when?

It is well known (see MZ07, Sect. 6.4]) that the answer to the first part of this question is "yes". In Theorem 4.1.9 we precisely characterize which tropical curves have the same Jacobian variety. In particular, we prove that for curves whose associated graph is 3-connected, the Torelli theorem holds in strong form, i.e. two such curves are tropically equivalent if and only if their polarized Jacobians are isomorphic.

The proof of Theorem 4.1.9 is based on a Torelli theorem for metric graphs, Theorem 4.1.10, which is interesting in its own right, and uses essentially the same ideas as the proof of Theorem 3.1.1. The statement of Theorem 4.1.10 is slightly more technical, but can be phrased as follows: two metric graphs have the same Albanese torus if and only if they have the same 3-edge connected class (defined in 2.3 .10 and 4.1.8).

A key ingredient turns out to be the Delaunay decomposition $\operatorname{Del}(\Gamma)$ of a graph $\Gamma$. $\operatorname{Del}(\Gamma)$ is well known to be a powerful tool, and has been investigated in, among others, Nam76, OS79 and Ale04, which have been quite useful in the writing of this paper. In Proposition 3.2.3 we characterize when two graphs have the same Delaunay decomposition.

The last section of the paper gives other characterizations of a graph, or rather, of the 3-edge connected class of a graph. These characterizations, given in Theorem 5.3.2, use three remarkable posets (i.e. partially ordered sets), $\mathcal{S} \mathcal{P}_{\Gamma}, \mathcal{O} \mathcal{P}_{\Gamma}$ and $\overline{\mathcal{O P}} \mathcal{P}_{\Gamma}$. The poset $\mathcal{S} \mathcal{P}_{\Gamma}$ is the set of spanning subgraphs of $\Gamma$ that are free from separating edges. The maximal elements of $\mathcal{S P}_{\Gamma}$ are the so-called C1-sets (see Definition 2.3.1), which play a crucial role in the previous sections. The two posets $\mathcal{O P} \mathcal{P}_{\Gamma}$ and $\overline{\mathcal{O P}} \mathcal{P}_{\Gamma}$, defined in Section 5.1, are associated to totally cyclic orientations; we conjecture a geometric interpretation for them in 5.2.8, relating to an interesting question posed in BdlHN97.

Not only is this last section related to the Torelli theorems in the previous parts, but also, our interest in it is motivated by a different, open, Torelli problem. The material of Section 5 will in fact be applied in our ongoing project, [CV], in order to describe the combinatorial structure of the compactified Jacobian of a singular algebraic curve, and generalize the Torelli theorem to stable curves.

In the Appendix, assuming some natural facts about the Torelli map $t_{g}^{\text {trop }}$ : $M_{g}^{\text {trop }} \rightarrow A_{g}^{\text {trop }}$ (facts that are commonly expected, yet still awaiting to be fully settled in the literature), we prove that $t_{g}^{\text {trop }}$ is of tropical degree one to its image, even though it is not injective; see Theorem A.2.1. This proves a conjecture of Mikhalkin-Zharkov (see [MZ07, Sect. 6.4]).

Acknowledgements. We thank L. Babai for a stimulating e-mail correspondence, M. Baker for pointing us the paper Art06, and G. Mikhalkin and I. Zharkov for precious comments on the tropical Torelli map, which prompted us to add the Appendix. The second author would like to thank T. Sunada for a series of lectures at Humboldt University of Berlin, during which he learnt about the Torelli problem for graphs, and G. Mikhalkin for a series of lectures at the INdAM workshop "Geometry of projective varieties", during which he learnt about the Torelli problem for tropical curves. Finally, we benefitted from a very thoughtful report by an anonymous referee, to whom we are grateful.

\section{Preliminaries}

2.1. The Albanese torus of a graph. Throughout the paper $\Gamma$ will be a finite graph (loops and multiple edges are allowed); we denote by $V(\Gamma)$ its set of vertices and by $E(\Gamma)$ its set of edges. 
We recall the definition of the Albanese torus, from KS00. Fix an orientation of $\Gamma$ and let $s, t: E(\Gamma) \rightarrow V(\Gamma)$ be the two maps sending an oriented edge to its source and target point, respectively. Notice that the Albanese torus will not depend on the chosen orientation. Consider the spaces of chains of $\Gamma$ with values in an abelian group $A$ :

$$
C_{0}(\Gamma, A):=\oplus_{v \in V(\Gamma)} A \cdot v, \quad C_{1}(\Gamma, A):=\oplus_{e \in E(\Gamma)} A \cdot e .
$$

Define, as usual, a boundary map

$$
\begin{aligned}
\partial: C_{1}(\Gamma, A) & \longrightarrow C_{0}(\Gamma, A) \\
e & \mapsto t(e)-s(e) .
\end{aligned}
$$

The first homology group of $\Gamma$ with values in $A$ is $H_{1}(\Gamma, A):=\operatorname{ker} \partial$.

If $A=\mathbb{R}$, we define the scalar product $($,$) on C_{1}(\Gamma, \mathbb{R})$ by

$$
\left(e, e^{\prime}\right)= \begin{cases}1 & \text { if } e=e^{\prime}, \\ 0 & \text { otherwise }\end{cases}
$$

We continue to denote by $($,$) the induced scalar product on H_{1}(\Gamma, \mathbb{R})$. The subspace $H_{1}(\Gamma, \mathbb{Z})$ is a lattice inside $H_{1}(\Gamma, \mathbb{R})$.

Definition 2.1.1. KS00 The Albanese torus $\mathrm{Alb}(\Gamma)$ of $\Gamma$ is

$$
\operatorname{Alb}(\Gamma):=\left(H_{1}(\Gamma, \mathbb{R}) / H_{1}(\Gamma, \mathbb{Z}) ;(,)\right)
$$

with the flat metric derived from the scalar product $($,$) .$

We have $\operatorname{dim} \operatorname{Alb}(\Gamma)=b_{1}(\Gamma)$ where $b_{1}(\Gamma)$ is the first Betti number:

$b_{1}(\Gamma)=\operatorname{rank}_{\mathbb{Z}} H_{1}(\Gamma, \mathbb{Z})=\#\{$ connected components of $\Gamma\}-\# V(\Gamma)+\# E(\Gamma)$.

There is also the cohomological version of the previous construction, (we refer to KS00 for the details). One obtains another torus, called the Jacobian torus $\operatorname{Jac}(\Gamma)$, which has the following form $\operatorname{Jac}(\Gamma):=\left(H^{1}(\Gamma, \mathbb{R}) / H^{1}(\Gamma, \mathbb{Z}) ;\langle\rangle,\right)$. As we said, $\operatorname{Jac}(\Gamma)$ and $\operatorname{Alb}(\Gamma)$ are dual flat tori.

There exist in the literature several definitions of Albanese and Jacobian torus of a graph, related to one another by means of standard dualities. In particular, we need to briefly explain the relation with [BdlHN97. Our lattice $H^{1}(\Gamma, \mathbb{Z})$ is the dual lattice, in $\left(H^{1}(\Gamma, \mathbb{R}) ;\langle\rangle,\right)$, of the so-called lattice of integral flows $\Lambda^{1}(\Gamma) \subset H^{1}(\Gamma, \mathbb{R})$ studied in [BdlHN97]. In particular, the Albanese torus $\operatorname{Alb}(\Gamma)$ determines the lattice $\Lambda^{1}(\Gamma)$ and conversely (see Proposition 3 of loc.cit.).

\subsection{Cyclic equivalence and connectivity.}

2.2.1. We set some notation that will be used throughout. Let $S \subset E(\Gamma)$ be a subset of edges of a graph $\Gamma$. We associate to $S$ two graphs, denoted $\Gamma \backslash S$ and $\Gamma(S)$, as follows

- The graph $\Gamma \backslash S$ is, as the notation indicates, obtained from $\Gamma$ by removing the edges in $S$ and by leaving the vertices unchanged. Thus $V(\Gamma \backslash S)=V(\Gamma)$ (so that $\Gamma \backslash S$ is a spanning subgraph) and $E(\Gamma \backslash S)=E(\Gamma) \backslash S$.

- The graph $\Gamma(S)$ is obtained from $\Gamma$ by contracting all the edges not in $S$, so that the set of edges of $\Gamma(S)$ is equal to $S$. There is a surjective contraction map $\Gamma \rightarrow \Gamma(S)$ which contracts to a point every connected component of $\Gamma \backslash S$. Notice that $\Gamma(S)$ is connected if and only if so is $\Gamma$. For example, $\Gamma(E(\Gamma))=\Gamma$, and, if $c$ is the number of connected components of $\Gamma$, then $\Gamma(\emptyset)$ is a set of $c$ isolated points (i.e. $\Gamma(\emptyset)$ has $c$ vertices and no edges).

Example 2.2.2. Here is an example of a $\Gamma(S)$, with the contraction map $\Gamma \rightarrow \Gamma(S)$, where $S=\left\{e_{1}, e_{2}\right\} \subset E(\Gamma)$ : 


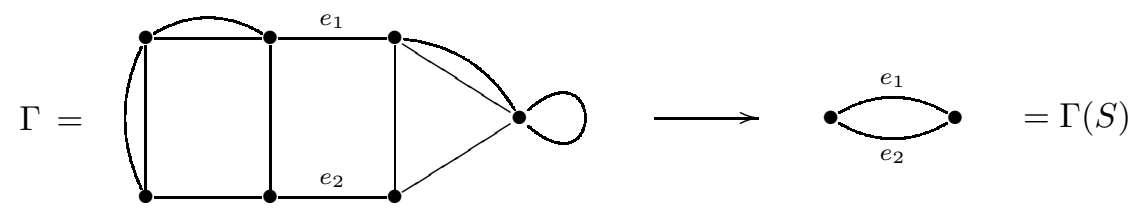

Figure 1. Example of $\Gamma(S)$ with $S=\left\{e_{1}, e_{2}\right\}$.

We have the useful additive formula

$$
b_{1}(\Gamma)=b_{1}(\Gamma \backslash S)+b_{1}(\Gamma(S)) .
$$

If $\Gamma$ is a connected graph, a separating edge is an $e \in E(\Gamma)$ such that $\Gamma \backslash e$ is not connected. If $\Gamma$ is not connected we say that an edge is separating if it is separating for the connected component containing it. We denote by $E(\Gamma)_{\text {sep }}$ the set of separating edges of $\Gamma$.

We say that a graph $\Delta$ is a cycle if it is connected, free from separating edges and if $b_{1}(\Delta)=1$. We call $\# E(\Delta)=\# V(\Delta)$ the length of $\Delta$.

Definition 2.2.3. Let $\Gamma$ and $\Gamma^{\prime}$ be two graphs. We say that a bijection between their edges, $\epsilon: E(\Gamma) \rightarrow E\left(\Gamma^{\prime}\right)$, is cyclic if it induces a bijection between the cycles of $\Gamma$ and the cycles of $\Gamma^{\prime}$.

We say that $\Gamma$ and $\Gamma^{\prime}$ are cyclically equivalent or 2-isomorphic, and we write $\Gamma \equiv_{\text {cyc }} \Gamma^{\prime}$, if there exists a cyclic bijection $\epsilon: E(\Gamma) \rightarrow E\left(\Gamma^{\prime}\right)$.

The cyclic equivalence class of $\Gamma$ will be denoted by $[\Gamma]_{\text {cyc }}$.

$[\Gamma]_{\text {cyc }}$ is described by the following result of Whitney (see also [Ox192, Sec. 5.3]).

Theorem 2.2.4 (Whi33]). Two graphs $\Gamma$ and $\Gamma^{\prime}$ are cyclically equivalent if and only if they can be obtained from one another via iterated applications of the following two moves:

(1) Vertex gluing: $v_{1}$ and $v_{2}$ are identified to the separating vertex $v$, and conversely (so that $\Gamma_{1} \amalg \Gamma_{2} \equiv_{\text {cyc }} \Gamma$ ).
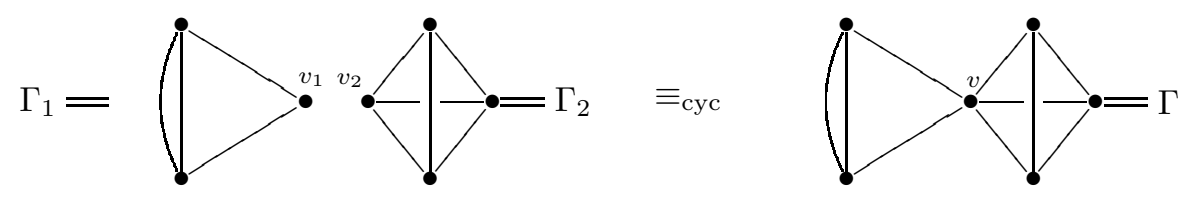

Figure 2. Two graphs $\Gamma_{1}$ and $\Gamma_{2}$ attached at $v_{1} \in V\left(\Gamma_{1}\right)$ and $v_{2} \in V\left(\Gamma_{2}\right)$.

(2) Twisting: the double arrows below mean identifications.
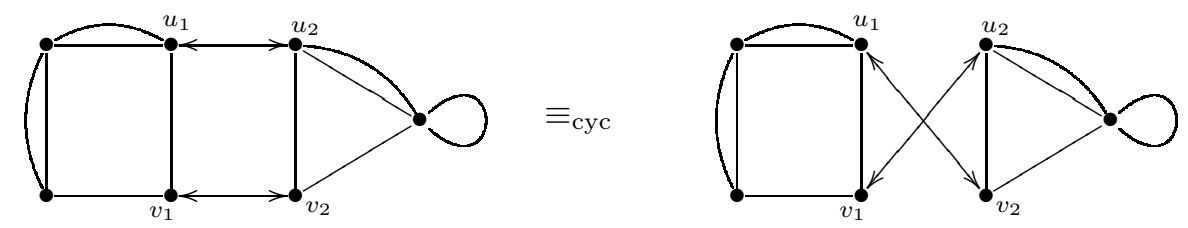

FIGURE 3. A twisting at a separating pair of vertices. 
Let us describe the above twisting move more precisely. Let $u, v$ be a pair of separating vertices of $\Gamma$. Then $\Gamma$ is obtained from two graphs, $\Gamma_{1}$ and $\Gamma_{2}$, by identifying two pairs of vertices as follows: Let $u_{i}, v_{i} \in V\left(\Gamma_{i}\right)$ for $i=1,2$. Then $\Gamma$ is given by attaching $\Gamma_{1}$ to $\Gamma_{2}$ by the two identifications $u_{1}=u_{2}=u$ and $v_{1}=v_{2}=u$. The twisting at the pair $u, v$ is the graph $\Gamma^{\prime}$ obtained by attaching $\Gamma_{1}$ to $\Gamma_{2}$ by the two identifications $u_{1}=v_{2}$ and $v_{1}=u_{2}$.

We now recall the definitions of connectivity (see for example [Die97, Chap. 3]). Let $k \geq 1$ be an integer. A graph $\Gamma$ having at least $k+1$-vertices is said to be $k$-connected if the graph obtained from $\Gamma$ by removing any $k-1$ vertices, and all the edges adjacent to them, is connected. A graph $\Gamma$ having at least 2 -vertices is said to be $k$-edge connected if the graph obtained from $\Gamma$ by removing any $k-1$ edges is connected.

If $\Gamma$ is $k$-connected it is also $k$-edge connected, but the converse fails.

$\Gamma$ is 1-connected, or 1-edge connected, if and only if it is connected.

$\Gamma$ is 2-edge connected if and only if it is connected and $E(\Gamma)_{\text {sep }}=\emptyset$.

3 -edge connected graphs will play an important role, and will be characterized in Corollary 2.3.4

Remark 2.2.5. We shall frequently consider edge-contracting maps, for which we make the following useful observation. Let $\Gamma \rightarrow \Gamma^{\prime}$ be a (surjective) map contracting some edge of $\Gamma$ to a point. Then if $\Gamma$ is $k$-edge connected so is $\Gamma^{\prime}$.

Remark 2.2.6. If $\Gamma$ is 3-connected, the cyclic equivalence class of $\Gamma$ contains only $\Gamma$. Indeed, by Theorem 2.2 .4 a move of type (1) can be performed only in the presence of a disconnecting vertex, and a move of type (2) in the presence of a separating pair of vertices.

\subsection{C1-sets and connectivizations.}

Definition 2.3.1. Let $\Gamma$ be a graph and $S \subset E(\Gamma)$.

Suppose $\Gamma$ connected and $E(\Gamma)_{\text {sep }}=\emptyset$; we say that $S$ is a $C 1$-set of $\Gamma$ if $\Gamma(S)$ is a cycle and if $\Gamma \backslash S$ has no separating edge.

In general, let $\widetilde{\Gamma}:=\Gamma \backslash E(\Gamma)_{\text {sep }}$. We say that $S$ is a C1-set of $\Gamma$ if $S$ is a C1-set of a connected component of $\widetilde{\Gamma}$.

We denote by $\operatorname{Set}^{1} \Gamma$ the set of $\mathrm{C} 1$-sets of $\Gamma$.

For instance, the set $S$ in Example 2.2.2 is a C1-set.

The terminology "C1" stands for "Codimension 1", and will be justified in 5.1.9. The following Lemma summarizes some useful properties of C1-sets.

Lemma 2.3.2. Let $\Gamma$ be a graph and $e, e^{\prime} \in E(\Gamma)$. Then

(i) Every C1-set $S$ of $\Gamma$ satisfies $S \cap E(\Gamma)_{\text {sep }}=\emptyset$.

(ii) Every non-separating edge e of $\Gamma$ is contained in a unique C1-set, $S_{e}$. If $E(\Gamma)_{\text {sep }}=\emptyset$, then $S_{e}=E(\Gamma \backslash e)_{\text {sep }} \cup\{e\}$.

(iii) $e$ and $e^{\prime}$ belong to the same C1-set if and only if they belong to the same cycles of $\Gamma$.

(iv) Assume $\Gamma$ connected and $e$ and $e^{\prime}$ non-separating. Then $e$ and $e^{\prime}$ belong to the same C1-set if and only if $\Gamma \backslash\left\{e, e^{\prime}\right\}$ is disconnected $\left(\left(e, e^{\prime}\right)\right.$ is called a separating pair of edges).

Proof. The first assertion follows trivially from Definition 2.3.1.

Notice that a C1-set of $\Gamma$ is entirely contained in the set of edges of a unique connected component of $\widetilde{\Gamma}$. Therefore we can assume that $\Gamma$ is connected, and, for parts (ii) and (iv), free from separating edges. 
Fix an edge $e \in E(\Gamma)$, let $\Gamma_{e}=\Gamma \backslash e$ and set

$$
S_{e}:=E\left(\Gamma_{e}\right)_{\operatorname{sep}} \cup\{e\} \subset E(\Gamma) .
$$

We claim that $S_{e}$ is the unique C1-set containing $e$. We have that $\Gamma\left(S_{e}\right)$ is connected and free from separating edges (as $\Gamma$ is). Therefore to prove that $S_{e}$ is a C1-set it suffices to prove that $b_{1}\left(\Gamma\left(S_{e}\right)\right)=1$. Let $\Gamma^{\prime}$ be the graph obtained from $\Gamma\left(S_{e}\right)$ by removing $e$; then $b_{1}\left(\Gamma^{\prime}\right)=0$ (by construction all its edges are separating). Now, $\# E\left(\Gamma\left(S_{e}\right)\right)=\# E\left(\Gamma^{\prime}\right)+1$, and, of course, $\Gamma\left(S_{e}\right)$ and $\Gamma^{\prime}$ have the same vertices. Therefore $b_{1}\left(\Gamma\left(S_{e}\right)\right)=b_{1}\left(\Gamma^{\prime}\right)+1=1$. So $S$ is a C1-set.

Finally, let $\widetilde{S}$ be a C1-set containing $e$. It is clear that $S_{e} \subset \widetilde{S}$ (any $e^{\prime} \in S_{e}$ such that $e^{\prime} \notin \widetilde{S}$ would be a separating edge of $\Gamma \backslash \widetilde{S}$ ). To prove that $S_{e}=\widetilde{S}$ consider the map $\Gamma \rightarrow \Gamma(\widetilde{S})$ contracting all the edges not in $\widetilde{S}$. Suppose, by contradiction, that there is an edge $\widetilde{e} \in \widetilde{S} \backslash S_{e}$; since $\Gamma(\widetilde{S})$ is a cycle, $\widetilde{e}$ is a separating edge of $\Gamma(\widetilde{S}) \backslash e$. Therefore $\widetilde{e}$ is a separating edge of $\Gamma \backslash e=\Gamma_{e}$, and hence $\widetilde{e}$ must lie in $S_{e}$, by 2.2. This is a contradiction, (iii) is proved.

Now part (iii). We can assume that $e$ and $e^{\prime}$ are non-separating, otherwise it is obvious. Suppose $S_{e}=S_{e^{\prime}}$; then, by definition, we can assume that $E(\Gamma)_{\text {sep }}=\emptyset$. Let $\Delta \subset \Gamma$ be a cycle containing $e^{\prime}$. By part (iii) we have that $e^{\prime}$ is a separating edge of $\Gamma \backslash e$; therefore if $\Delta$ does not contain $e$, then $e^{\prime}$ is a separating edge of $\Delta$, which is impossible. Conversely, if $e^{\prime} \notin S_{e}$ then (as $e^{\prime}$ is non-separating for $\Gamma \backslash S_{e}$ ) there exists a cycle $\Delta \subset \Gamma \backslash S_{e}$ containing $e^{\prime}$. So $e$ and $e^{\prime}$ do not lie in the same cycles.

Finally part (iv). If $\left(e, e^{\prime}\right)$ is a separating pair then $e$ is a separating edge of $\Gamma \backslash e^{\prime}$ and $e^{\prime}$ is a separating edge of $\Gamma \backslash e$. By part (iii) $e$ and $e^{\prime}$ belong to the same C1-set. The converse follows from the fact that a cycle with two edges removed is disconnected.

Remark 2.3.3. Let $\Delta \subset \Gamma$ be a cycle. By Lemma 2.3 .2 the set $E(\Delta)$ is a disjoint union of C1-sets. We define $\operatorname{Set}_{\Delta}^{1} \Gamma:=\left\{S \in \operatorname{Set}^{1} \Gamma: S \subset E(\Delta)\right\}$ so that

$$
E(\Delta)=\coprod_{S \in \operatorname{Set}_{\Delta}^{1} \Gamma} S
$$

Corollary 2.3.4. A graph $\Gamma$ is 3-edge connected if and only if it is connected and there is a bijection $E(\Gamma) \rightarrow \operatorname{Set}^{1} \Gamma$ mapping $e \in E(\Gamma)$ to $\{e\} \in \operatorname{Set}^{1} \Gamma$.

Proof. If $\Gamma$ is 3-edge connected it is free from separating edges; hence every $e \in E(\Gamma)$ belongs to a unique $S \in \operatorname{Set}^{1} \Gamma$. So it suffices to prove that every $S \in \operatorname{Set}^{1} \Gamma$ has cardinality 1. Suppose there are two distinct edges $e, e^{\prime} \in S$. Then Lemma 2.3.2(iv) yields that $\Gamma \backslash\left\{e, e^{\prime}\right\}$ is not connected, which is a contradiction.

Conversely, if every edge lies in a $\mathrm{C} 1$-set, then $\Gamma$ has no separating edges. If $\Gamma$ is not 3 -edge connected, it admits a separating pair of edges $\left(e, e^{\prime}\right)$. Then $e$ and $e^{\prime}$ belong to the same $S \in \operatorname{Set}^{1} \Gamma$ (by Lemma 2.3.2). So we are done.

In the next statement we use the notation of 2.3.2 (iii) and 2.2.3.

Corollary 2.3.5. Let $\Gamma$ and $\Gamma^{\prime}$ be cyclically equivalent; then $\# E(\Gamma)_{\text {sep }}=\# E\left(\Gamma^{\prime}\right)_{\text {sep }}$. Let $\epsilon: E(\Gamma) \rightarrow E\left(\Gamma^{\prime}\right)$ be a cyclic bijection; then $\epsilon$ induces a bijection

$$
\begin{aligned}
\beta_{\epsilon}: \operatorname{Set}^{1} \Gamma & \longrightarrow \operatorname{Set}^{1} \Gamma^{\prime} \\
S_{e} & \longmapsto S_{\epsilon(e)}
\end{aligned}
$$

such that $\# S=\# \beta_{\epsilon}(S)$ for every $S \in \operatorname{Set}^{1} \Gamma$.

Proof. An edge is separating if and only if it is not contained in any cycle. Therefore $\epsilon$ maps $E(\Gamma)_{\text {sep }}$ bijectively to $E\left(\Gamma^{\prime}\right)_{\text {sep }}$, so the first part is proved. The second part follows immediately from Lemma 2.3.2 (iii) and (iii). 
We introduce two types of edge contractions that will be used extensively later:

(A) Contraction of a separating edge:

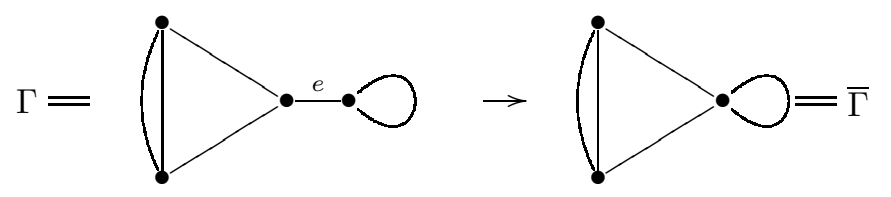

FigurE 4. The contraction of the separating edge $e \in E(\Gamma)$.

(B) Contraction of one of two edges of a separating pair of edges:

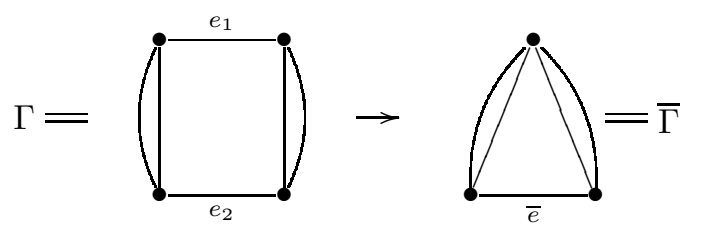

FigurE 5. The contraction of the edge $e_{1}$ of the separating pair $\left(e_{1}, e_{2}\right)$.

To a graph $\Gamma$ we shall associate two types of graphs.

Definition 2.3.6. The 2-edge connectivization of a connected graph $\Gamma$ is the 2 -edge connected graph $\Gamma^{2}$ obtained from $\Gamma$ by iterating the above operation (A) (for all the separating edges of $\Gamma$ ).

A 3-edge connectivization of a connected graph $\Gamma$ is a 3-edge connected graph $\Gamma^{3}$ which is obtained from $\Gamma^{2}$ by iterating the above operation (B).

If $\Gamma$ is not connected, we define $\Gamma^{2}$ (resp. $\Gamma^{3}$ ) as the disjoint union of the 2-edge connectivizations (resp. 3-edge connectivizations) of its connected components.

Remark 2.3.7. It is clear that $\Gamma^{2}$ is uniquely determined, while $\Gamma^{3}$ is not.

If $\Gamma$ is not connected $\Gamma^{2}$ (resp. $\Gamma^{3}$ ) is not 2-edge (resp. 3-edge) connected.

There is a (surjective) contraction map $\sigma: \Gamma \rightarrow \Gamma^{2} \rightarrow \Gamma^{3}$ obtained by composing the contractions defining $\Gamma^{2}$ and $\Gamma^{3}$.

Lemma 2.3.8. Let $\Gamma$ be a graph.

(i) $b_{1}\left(\Gamma^{3}\right)=b_{1}\left(\Gamma^{2}\right)=b_{1}(\Gamma)$.

(ii) There are canonical bijections

$$
\operatorname{Set}^{1} \Gamma^{3} \leftrightarrow E\left(\Gamma^{3}\right) \leftrightarrow \operatorname{Set}^{1} \Gamma .
$$

(iii) Two 3-edge connectivizations of $\Gamma$ are cyclically equivalent.

(iv) $\Gamma^{2} \equiv_{\text {cyc }} \Gamma \backslash E(\Gamma)_{\text {sep }}$.

Proof. The first Betti number is invariant under the operations (A) and (B) above, because no loop gets contracted. So, part (ii) is done. Notice also (which will be used later) that the contraction map $\sigma: \Gamma \rightarrow \Gamma^{3}$ induces a natural bijection between the cycles of $\Gamma$ and those of $\Gamma^{3}$.

Now part (iii). The bijection $\operatorname{Set}^{1} \Gamma^{3} \leftrightarrow E\left(\Gamma^{3}\right)$ is described in 2.3.4 Let $S \in \operatorname{Set}^{1} \Gamma$ and set

$$
S=\left\{e_{S, 1}, \ldots, e_{S, \# S}\right\}
$$


Consider again the contraction map $\sigma: \Gamma \rightarrow \Gamma^{3}$. Clearly $\sigma$ contracts all the edges of $S$ but one, which gets mapped to an edge $e_{S} \in E\left(\Gamma^{3}\right)$. We have thus defined a map

$$
\psi: \operatorname{Set}^{1} \Gamma \longrightarrow E\left(\Gamma^{3}\right) ; \quad S \longmapsto e_{S} .
$$

By 2.3.2 and by the definition of $\Gamma^{3}$ the above map is a bijection. So (iii) is proved.

Let $\Gamma^{3}$ and $\widetilde{\Gamma^{3}}$ be two 3-edge connectivizations of $\Gamma$. By part (iii) there is a natural bijection $E\left(\Gamma^{3}\right) \leftrightarrow E\left(\widetilde{\Gamma^{3}}\right)$. Moreover, by what we said before, the two contraction maps

$$
\sigma: \Gamma \longrightarrow \Gamma^{3} \quad \tilde{\sigma}: \Gamma \longrightarrow \widetilde{\Gamma^{3}}
$$

induce natural bijections between cycles that are compatible with the bijection $E\left(\Gamma^{3}\right) \leftrightarrow E\left(\widetilde{\Gamma^{3}}\right)$. Therefore $\Gamma^{3} \equiv_{\text {cyc }} \widetilde{\Gamma^{3}}$, and the part (iii) is proved.

For the last part, it suffices to observe that $\Gamma^{2}$ can be obtained by $\Gamma \backslash E(\Gamma)_{\text {sep }}$ by moves of type (1) (vertex gluing) in Theorem 2.2.4.

Proposition 2.3.9. Let $\Gamma$ and $\Gamma^{\prime}$ be two graphs.

(i) Assume $\Gamma^{2} \equiv_{\text {cyc }} \Gamma^{\prime 2}$. Then $\Gamma \equiv_{\text {cyc }} \Gamma^{\prime}$ if and only if $\# E(\Gamma)_{\text {sep }}=\# E\left(\Gamma^{\prime}\right)_{\text {sep }}$.

(ii) Assume $\Gamma^{3} \equiv_{\text {cyc }} \Gamma^{\prime 3}$ and $E(\Gamma)_{\text {sep }}=E\left(\Gamma^{\prime}\right)_{\text {sep }}=\emptyset$. Then $\Gamma \equiv_{\text {cyc }} \Gamma^{\prime}$ if and only if the natural bijection

$$
\beta: \operatorname{Set}^{1} \Gamma \stackrel{\psi}{\longrightarrow} E\left(\Gamma^{3}\right) \stackrel{\epsilon^{3}}{\longrightarrow} E\left(\Gamma^{\prime 3}\right) \stackrel{\left(\psi^{\prime}\right)^{-1}}{\longrightarrow} \operatorname{Set}^{1} \Gamma^{\prime}
$$

satisfies $\# S=\# \beta(S)$, where $\psi$ and $\psi^{\prime}$ are the bijections defined in 2.4), and $\epsilon^{3}$ a cyclic bijection.

Proof. The "only if" part for both (ii) and (iii) holds in general, by Corollary 2.3.5] It suffices to add (for part (iii)) that any cyclic bijection $\epsilon: E(\Gamma) \rightarrow E\left(\Gamma^{\prime}\right)$ induces a canonical cyclic bijection $\epsilon^{3}: E\left(\Gamma^{3}\right) \rightarrow E\left(\Gamma^{\prime 3}\right)$, and it is clear that $\left(\psi^{\prime}\right)^{-1} \circ \epsilon^{3} \circ \psi=\beta_{\epsilon}$ defined in 2.3.5.

Let us prove the sufficiency for part (ii). The point is that we can identify the edges of $\Gamma^{2}$ with the non-separating edges of $\Gamma$ so that we have $E(\Gamma)=$ $E\left(\Gamma^{2}\right) \coprod E(\Gamma)_{\text {sep }}$; the same holds for $\Gamma^{\prime}$ of course. So, pick a cyclic bijection $\epsilon^{2}: E\left(\Gamma^{2}\right) \rightarrow E\left(\Gamma^{\prime 2}\right)$ and any bijection $\epsilon_{\text {sep }}: E(\Gamma)_{\text {sep }} \rightarrow E\left(\Gamma^{\prime}\right)_{\text {sep }}$. Then we can glue $\epsilon^{2}$ with $\epsilon_{\text {sep }}$ to a bijection $\epsilon: E(\Gamma) \rightarrow E\left(\Gamma^{\prime}\right)$ which is easily seen to be cyclic.

Now we prove the sufficiency in part (iii). Recall that the contraction map $\sigma$ : $\Gamma \rightarrow \Gamma^{3}$ induces a natural bijection between the cycles of $\Gamma$ and the cycles of $\Gamma^{3}$; and the same holds for $\Gamma^{\prime}$. Therefore $\epsilon^{3}$ induces a bijection, call it $\eta$, between the cycles of $\Gamma$ and the cycles of $\Gamma^{\prime}$.

On the other hand the bijection $\beta$ in the statement induces a (non unique) bijection between $\epsilon: E(\Gamma) \rightarrow E\left(\Gamma^{\prime}\right)$. Indeed, as $\Gamma$ and $\Gamma^{\prime}$ have no separating edges, every edge belongs to a unique $\mathrm{C} 1$-set (2.3.2). As $\beta$ preserves the cardinality of the $\mathrm{C} 1$-sets, we easily obtain our $\epsilon$. To show that $\epsilon$ is cyclic, it suffices to observe that, because of the naturality of the various maps, $\epsilon$ induces the above bijection $\eta$ between cycles of $\Gamma$ and $\Gamma^{\prime}$.

Remark 2.3.10. By the previous results, the class $\left[\Gamma^{3}\right]_{\text {cyc }}$ depends solely on $[\Gamma]_{\text {cyc }}$.

Moreover, every representative in the class $\left[\Gamma^{3}\right]_{\text {cyc }}$ is such that each of its connected components is 3 -edge-connected; therefore we shall refer to $\left[\Gamma^{3}\right]_{\mathrm{cyc}}$ as the 3-edge connected class of $\Gamma$.

\subsection{Totally cyclic orientations.}

Definition 2.4.1. Let $\Gamma$ be a graph and $V(\Gamma)$ its set of vertices.

If $\Gamma$ is connected, we say that an orientation of $\Gamma$ is totally cyclic if there exists no proper non-empty subset $W \subset V(\Gamma)$ such that the edges between $W$ and its 
complement $V(\Gamma) \backslash W$ go all in the same direction. i.e. either all from $W$ to $V(\Gamma) \backslash W$, or all in the opposite direction.

If $\Gamma$ is not connected, we say that an orientation of $\Gamma$ is totally cyclic if the orientation induced on each connected component of $\Gamma$ is totally cyclic.

Other names for these orientations are "strongly connected", and "stable" (the latter is used in algebraic geometry).

Remark 2.4.2. A cycle $\Delta$ admits exactly two totally cyclic orientations, which are usually called just cyclic, for obvious reasons.

On the other hand if $E(\Gamma)_{\text {sep }} \neq \emptyset$ then $\Gamma$ admits no totally cyclic orientations. Indeed, suppose $\Gamma$ connected for simplicity and let $e \in E(\Gamma)_{\text {sep }}$. Then the graph $\Gamma \backslash e$ is the disjoint union of two graphs $\Gamma_{1}$ and $\Gamma_{2}$. Then the set $W=V\left(\Gamma_{1}\right) \subset V(\Gamma)$ does not satisfy the requirement of Definition 2.4.1

The following lemma, the first part of which is already known, will be very useful.

Lemma 2.4.3. Let $\Gamma$ be a graph.

(1) $\Gamma$ admits a totally cyclic orientation if and only if $E(\Gamma)_{\operatorname{sep}}=\emptyset$.

(2) Assume $E(\Gamma)_{\mathrm{sep}}=\emptyset$ and fix an orientation on $\Gamma$. The following conditions are equivalent:

(a) The orientation is totally cyclic.

(b) For any distinct $v, w \in V(\Gamma)$ belonging to the same connected component of $\Gamma$, there exists a path oriented from $w$ to $v$.

(c) $H_{1}(\Gamma, \mathbb{Z})$ has a basis of cyclically oriented cycles.

(d) Every edge $e \in E(\Gamma)$ is contained in a cyclically oriented cycle.

Proof. Part (1). We already observed, in 2.4.2, that if $\Gamma$ has a separating edge it does not admit a totally cyclic orientation. The converse, which is the nontrivial part, was proved in Rob39, or later in [Cap08, Lemma 1.3.5].

We now prove the equivalence of the four conditions in Part (2).

(2a) $\Rightarrow(2 \mathrm{~b})$ Pick $w \in V(\Gamma)$. Let $W \subset V(\Gamma)$ be the set of all vertices $v$ such that $\Gamma$ contains a path oriented from $w$ to $v$. We want to prove that $W=V(\Gamma)$. By contadiction, suppose that $V(\Gamma) \backslash W$ is not empty. Then every edge $e$ joining a vertex $w^{\prime}$ in $W$ with a vertex $v$ in $V(\Gamma) \backslash W$ must be oriented from $v$ to $w^{\prime}$ (otherwise the path obtained attaching $e$ to an oriented path from $w$ to $w^{\prime}$ would be oriented from $w$ to $v$, so that $v \in W$, which is not the case). But then every edge between $V(\Gamma) \backslash W$ and $W$ goes from the former to the latter, hence the orientation is not totally cyclic, a contradiction.

$(2 \mathrm{~b}) \Rightarrow(2 \mathrm{c})$. Let $d \in H_{1}(\Gamma, \mathbb{Z})$ be an element corresponding to a cycle $\Delta$. We claim that $d$ can be expressed as $d=\sum n_{i} d_{i}$ with each $d_{i}$ corresponding to a cyclically oriented cycle $\Delta_{i} \subset \Gamma$, and $n_{i} \in \mathbb{Z}$. Suppose that $\Delta$ is not cyclically oriented (otherwise there is nothing to prove). Clearly every edge of $\Delta$ is contained in a unique maximal oriented (connected) path contained in $\Delta$. This enables us to express $\Delta$ as a union of maximal oriented paths, call them $p_{1}, \ldots, p_{c}$, such that every $p_{i}$ is adjacent to $p_{i-1}$ and $p_{i+1}$ (with the cyclic convention $p_{0}=p_{c}$; note that $c \geq 2$ ). More precisely, call $v_{1}, \ldots, v_{c}$ the vertices of this decomposition, so that $v_{i}, v_{i+1}$ are the end points of $p_{i}$ for every $i<c$ and $v_{c}, v_{1}$ are the end points of $p_{c}$. Call $s_{i}$, respectively $t_{i}$, the starting, respectively the ending, vertex of each path. With no loss of generality, we may assume that $s_{1}=v_{1}$ (i.e. $p_{1}$ starts from $v_{1}$ ) and that $d=\sum_{i=1}^{c}(-1)^{i-1} p_{i}$ (abusing notation slightly). By the maximality assumption, we obtain that every odd vertex is the source of both its adjacent paths, and every even vertex is the target of its adjacent paths, i.e.:

$$
v_{2 i+1}=s_{2 i}=s_{2 i+1}, \quad v_{2 i}=t_{2 i}=t_{2 i-1} .
$$


Notice that the number of paths, $c$, is necessarily even.

Now, by (2b) we can pick a set of paths, $q_{1}, \ldots, q_{c-1}$, in $\Gamma$ such that $q_{i}$ joins $v_{1}$ and $v_{i+1}$ and is oriented as follows. For every odd $i$ the path $q_{i}$ starts from $v_{i+1}$ and ends in $v_{1}$. For every even $i$ the path $q_{i}$ starts from $v_{1}$ and ends in $v_{i+1}$.

With this choice, we have the following cyclically oriented cycles $\Delta_{1}, \ldots \Delta_{c}$. The cycle $\Delta_{1}$ is obtained by composing the paths $p_{1}$ and $q_{1}$; for all $1<i<c$ the cycle $\Delta_{i}$ is obtained by composing the paths $p_{i}, q_{i}$ and $q_{i-1}$; finally $\Delta_{c}$ is the composition of $p_{c}$ with $q_{c-1}$. We have

$d=\sum_{i=1}^{c}(-1)^{i-1} p_{i}=p_{1}+q_{1}-\sum_{i=2}^{c-1}(-1)^{i}\left(p_{i}+q_{i}+q_{i-1}\right)-p_{c}-q_{c-1}=\sum_{i=1}^{c}(-1)^{i-1} d_{i}$

where $d_{i} \in H_{1}(\Gamma, \mathbb{Z})$ corresponds to $\Delta_{i}$. This proves that the $\mathbb{Z}$-span of the set of cyclically oriented cycles is the entire $H_{1}(\Gamma, \mathbb{Z})$.

$(2 \mathrm{c}) \Rightarrow(2 \mathrm{~d})$. Pick a basis of cyclically oriented cycles for $H_{1}(\Gamma, \mathbb{Z})$. By contradiction, let $e \in E(\Gamma)$ be such that there exists no cyclically oriented cycle containing it. Then there exists no basis element containing $e$, and hence $e$ is not contained in any cycle, which is obviously impossible (as $\left.E(\Gamma)_{\text {sep }}=\emptyset\right)$.

$(2 \mathrm{~d}) \Rightarrow(2 \mathrm{a})$. By contradiction, assume there exists a set of vertices $W$ such that $\emptyset \subsetneq W \subsetneq V(\Gamma)$ and such that every edge between $W$ and $V(\Gamma) \backslash W$ goes from $W$ to $V(\Gamma) \backslash W$. Let $e$ be any such edge; every cycle $\Delta$ containing $e$ must contain another edge $e^{\prime}$ between $W$ and $V(\Gamma) \backslash W$, and therefore (as $e^{\prime}$ is also oriented from $W$ to $V(\Gamma) \backslash W) \Delta$ is not cyclically oriented. We conclude that no cycle containing $e$ is cyclically oriented, and this contradicts part (2d).

We shall use the following notation. For any edge $e \in E(\Gamma)$, we denote by $e^{*} \in C_{1}(\Gamma, \mathbb{R})^{*}$ the functional on $C_{1}(\Gamma, \mathbb{R})$ defined, for $e^{\prime} \in E(\Gamma)$,

$$
e^{*}\left(e^{\prime}\right)= \begin{cases}1 & \text { if } e^{\prime}=e, \\ 0 & \text { otherwise. }\end{cases}
$$

We shall constantly abuse notation by calling $e^{*} \in H_{1}(\Gamma, \mathbb{R})^{*}$ also the restriction of $e^{*}$ to $H_{1}(\Gamma, \mathbb{R})$.

Remark 2.4.4. $e \in E(\Gamma)_{\text {sep }}$ if and only if the restriction of $e^{*}$ to $H_{1}(\Gamma, \mathbb{R})$ is zero.

Indeed $e \in E(\Gamma)_{\text {sep }}$ if and only if $e$ is not contained in any cycle of $\Gamma$.

Recall that for any $S \in \operatorname{Set}^{1} \Gamma$ we denote $S=\left\{e_{S, 1}, \ldots, e_{S, \# S}\right\}$.

Corollary 2.4.5. Let $\Gamma$ be a graph and fix an orientation inducing a totally cyclic orientation on $\Gamma \backslash E(\Gamma)_{\text {sep. }}$. Then the following facts hold.

(1) For every $c \in H_{1}(\Gamma, \mathbb{Z})$ we have

$$
c=\sum_{S \in \operatorname{Set}^{1} \Gamma} r_{S}(c) \sum_{i=1}^{\# S} e_{S, i}, \quad r_{S}(c) \in \mathbb{Z}
$$

(2) Let $e_{1}, e_{2} \in E(\Gamma) \backslash E(\Gamma)_{\mathrm{sep}}$. There exists $u \in \mathbb{R}$ such that $e_{1}^{*}=u e_{2}^{*}$ on $H_{1}(\Gamma, \mathbb{R})$ if and only if $e_{1}$ and $e_{2}$ belong to the same C1-set of $\Gamma$; moreover, in this case $u=1$.

Proof. Let $\Delta \subset \Gamma$ be a cyclically oriented cycle. Then $\sum_{e \in E(\Delta)} e \in H_{1}(\Gamma, \mathbb{Z})$. By Lemma 2.3.2 (iii), if a $\mathrm{C} 1$-set intersects the set of edges of a cycle, then it is entirely contained in it. So part (1) follows from Lemma 2.4.3 (2c).

For the second part, if $e_{1}$ and $e_{2}$ belong to the same C1-set then $e_{1}^{*}=e_{2}^{*}$ by the first part. Conversely, suppose $e_{1}$ and $e_{2}$ belong to different C1-sets, $S_{1}$ and $S_{2}$. Then by Lemma 2.3 .2 (iii) there exists a cycle containing $e_{1}$ and not $e_{2}$. 
Hence there exists $c \in H_{1}(\Gamma, \mathbb{Z})$ such that $r_{S_{1}}(c) \neq 0$ and $r_{S_{2}}(c)=0$. But then $e_{1}^{*}(c)=r_{S_{1}}(c) \neq 0$ and $e_{2}^{*}(c)=r_{S_{2}}(c)=0$ therefore $e_{1}^{*} \neq u e_{2}^{*}$ for any $u \in \mathbb{R}$.

\section{TORELLI THEOREM FOR GRAPHS}

3.1. Statement of the theorem. The aim of this section is to prove the following Torelli theorem for graphs.

Theorem 3.1.1. Let $\Gamma$ and $\Gamma^{\prime}$ be two graphs. Then $\operatorname{Alb}(\Gamma) \cong \operatorname{Alb}\left(\Gamma^{\prime}\right)$ if and only if $\Gamma^{2} \equiv_{\mathrm{cyc}} \Gamma^{\prime 2}$.

We deduce that the Torelli theorem is true in stronger form for 3-connected graphs. More generally:

Corollary 3.1.2. Let $\Gamma$ be 3 -connected and let $\Gamma^{\prime}$ have no vertex of valence 1 . Then $\operatorname{Alb}(\Gamma) \cong \operatorname{Alb}\left(\Gamma^{\prime}\right)$ if and only if $\Gamma \cong \Gamma^{\prime}$.

Proof. By hypothesis $\Gamma^{2}=\Gamma$. Assume $\operatorname{Alb}(\Gamma) \cong \operatorname{Alb}\left(\Gamma^{\prime}\right)$; then Theorem 3.1.1yields $\Gamma \equiv_{\text {cyc }} \Gamma^{\prime 2}$. By Remark 2.2.6 we obtain $\Gamma \cong \Gamma^{\prime 2}$. If $\Gamma^{\prime 2} \neq \Gamma^{\prime}$, then the contraction map $\Gamma^{\prime} \rightarrow \Gamma^{\prime 2}$ certainly produces some separating vertex, given by the image of a separating edge of $\Gamma^{\prime}$ (because $\Gamma^{\prime}$ has non vertex of valence 1). But $\Gamma^{\prime 2}$ has no such vertices, by the assumption on $\Gamma$. Hence we necessarily have $\Gamma^{\prime} \cong \Gamma^{\prime 2} \cong \Gamma$.

Proof of Theorem 3.1.1: sufficiency. The "if" direction of Theorem 3.1.1] is not difficult, and it follows from the subsequent statement, part (ii) of which is already known; see [BdlHN97, Prop. 5] (where a different language is used).

Proposition 3.1.3. Let $\Gamma$ be a graph.

(i) $\operatorname{Alb}(\Gamma)$ depends only on $[\Gamma]_{\mathrm{cyc}}$.

(ii) $\operatorname{Alb}(\Gamma)=\operatorname{Alb}\left(\Gamma^{2}\right)$.

Proof. Part (i) follows from the fact that $\left(H_{1}(\Gamma, \mathbb{Z}) ;(),\right)$ is defined entirely in terms of the inclusion $H_{1}(\Gamma, \mathbb{Z}) \subset C_{1}(\Gamma, \mathbb{Z})$ and of the basis $E(\Gamma)$ of $C_{1}(\Gamma, \mathbb{Z})$, which is clearly invariant by cyclic equivalence.

For the second part, first note that we can naturally identify

$$
E\left(\Gamma^{2}\right)=E(\Gamma) \backslash E(\Gamma)_{\text {sep }} \subset E(\Gamma) .
$$

We fix orientations on $\Gamma^{2}$ and $\Gamma$ that are compatible with respect to the above (3.1). It is clear that there is a natural commutative diagram

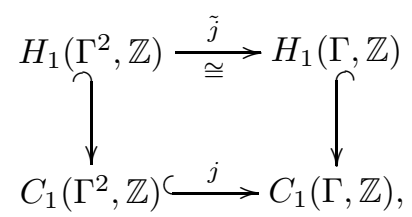

where the vertical maps are the inclusions, $j$ is induced by the inclusion (3.1), and $\tilde{j}$ denotes the restriction of $j$. Part (iii) follows from the diagram and the fact that the inclusion $j$ is compatible with the scalar products $($,$) on both sides.$

From Proposition 3.1 .3 we derive that if $\Gamma^{2} \equiv_{\mathrm{cyc}} \Gamma^{\prime 2}$ then $\operatorname{Alb}(\Gamma)=\operatorname{Alb}\left(\Gamma^{\prime}\right)$. Hence the sufficiency in Theorem 3.1.1 is proved.

In order to prove the other half of the theorem, we need some preliminaries. 
3.2. The Delaunay decomposition. Consider the lattice $H_{1}(\Gamma, \mathbb{Z})$ inside the real vector space $H_{1}(\Gamma, \mathbb{R})$. Observe that the scalar product induced on $C_{1}(\Gamma, \mathbb{R})$ by $($, coincides with the Euclidean scalar product. We denote the norm $\sqrt{(x, x)}$ by $\|x\|$.

Definition 3.2.1. For any $\alpha \in H_{1}(\Gamma, \mathbb{R})$, a lattice element $x \in H_{1}(\Gamma, \mathbb{Z})$ is called $\alpha$-nearest if

$$
\|x-\alpha\|=\min \left\{\|y-\alpha\|: y \in H_{1}(\Gamma, \mathbb{Z})\right\} .
$$

A Delaunay cell is defined as the closed convex hull of all elements of $H_{1}(\Gamma, \mathbb{Z})$ which are $\alpha$-nearest for some fixed $\alpha \in H_{1}(\Gamma, \mathbb{R})$. Together, all the Delaunay cells constitute a locally finite decomposition of $H_{1}(\Gamma, \mathbb{R})$ into infinitely many bounded convex polytopes, called the Delaunay decomposition of $\Gamma$, denoted $\operatorname{Del}(\Gamma)$.

Let $\Gamma$ and $\Gamma^{\prime}$ be two graphs. We say that $\operatorname{Del}(\Gamma) \cong \operatorname{Del}\left(\Gamma^{\prime}\right)$ if there exists a linear isomorphism $H_{1}(\Gamma, \mathbb{R}) \rightarrow H_{1}\left(\Gamma^{\prime}, \mathbb{R}\right)$ sending $H_{1}(\Gamma, \mathbb{Z})$ into $H_{1}\left(\Gamma^{\prime}, \mathbb{Z}\right)$ and mapping the Delaunay cells of $\operatorname{Del}(\Gamma)$ isomorphically into the Delaunay cells of $\operatorname{Del}\left(\Gamma^{\prime}\right)$.

Remark 3.2.2. It is well known that an equivalent, and for us very useful, definition is the following. The Delaunay decomposition $\operatorname{Del}(\Gamma)$ is the restriction to $H_{1}(\Gamma, \mathbb{R})$ of the decomposition of $C_{1}(\Gamma, \mathbb{R})$ consisting of the standard cubes cut out by all hyperplanes of equation $e^{*}=n$ for $e \in E(\Gamma)$ and $n \in \mathbb{Z}$; see OS79, Prop. 5.5]. These hyperplanes of $H_{1}(\Gamma, \mathbb{R})$, having equations $e^{*}=n$, are called the generating hyperplanes of the Delaunay decomposition. Notice that an isomorphism $\operatorname{Del}(\Gamma) \cong$ $\operatorname{Del}\left(\Gamma^{\prime}\right)$ induces a bijection between the sets of generating hyperplanes.

Proposition 3.2.3. Let $\Gamma$ and $\Gamma^{\prime}$ be two graphs.

(i) $\operatorname{Del}(\Gamma)$ depends only on $[\Gamma]_{\text {cyc }}$.

(ii) $\operatorname{Del}(\Gamma) \cong \operatorname{Del}\left(\Gamma^{3}\right)$ for any choice of $\Gamma^{3}$

(iii) $\operatorname{Del}(\Gamma) \cong \operatorname{Del}\left(\Gamma^{\prime}\right)$ if and only if $\Gamma^{3} \equiv_{\text {cyc }} \Gamma^{\prime 3}$.

Proof. It is clear that the Delaunay decomposition is completely determined by the inclusion $H_{1}(\Gamma, \mathbb{Z}) \subset C_{1}(\Gamma, \mathbb{Z})$ together with the basis $E(\Gamma)$ of $C_{1}(\Gamma, \mathbb{Z})$ defining the scalar product $($,$) . This proves part (ii).$

Let us now prove part (iii). First note that $\operatorname{Del}(\Gamma) \cong \operatorname{Del}\left(\Gamma^{2}\right)$, as it follows easily from diagram (3.2) and Remark 2.4.4. We can therefore assume that $\Gamma$ is 2-edge connected. Consider the natural bijection (cf. (2.4))

$$
\begin{aligned}
& \psi: \operatorname{Set}^{1} \Gamma \longrightarrow E\left(\Gamma^{3}\right) \\
& S \longmapsto \quad e_{S}
\end{aligned}
$$

where $e_{S}$ is the only edge in $S$ which is not contracted by the contraction map $\sigma: \Gamma \rightarrow \Gamma^{3}$. We can thus define an injection

$$
\begin{aligned}
C_{1}\left(\Gamma^{3}, \mathbb{Z}\right) \stackrel{\iota}{\longrightarrow} C_{1}(\Gamma, \mathbb{Z}) \\
e_{S} \longmapsto \sum_{i=1}^{\# S} e_{S, i},
\end{aligned}
$$

where for any $S \in \operatorname{Set}^{1} \Gamma$ we denote, as in (2.3), $S=\left\{e_{S, 1}, \ldots, e_{S, \# S}\right\}$. Fix now a totally cyclic orientation on $\Gamma$ and the induced orientation on $\Gamma^{3}$; consider the corresponding spaces $H_{1}(\Gamma, \mathbb{Z})$ and $H_{1}\left(\Gamma^{3}, \mathbb{Z}\right)$. We claim that the above injection induces a natural diagram

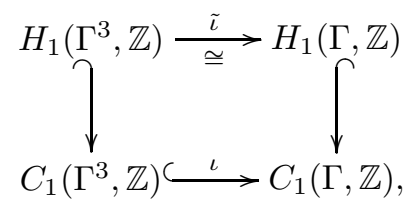


where the vertical maps are the inclusions, and $\tilde{\iota}$ is the restriction of $\iota$. Indeed, the image of $\iota$ is clearly the subset $K_{2} \subset C_{1}(\Gamma, \mathbb{Z})$ defined by

$$
K_{2}:=\bigcap_{S \in \operatorname{Set}^{1} \Gamma} \bigcap_{i, j=1}^{\# S} \operatorname{ker}\left(e_{S, i}^{*}-e_{S, j}^{*}\right) .
$$

Moreover, by Corollary 2.4.5 we get that $H_{1}(\Gamma, \mathbb{Z}) \subset K_{2}$. On the other hand, the contraction map $\sigma: \Gamma \rightarrow \Gamma^{3}$ induces a bijection between cycles, therefore $H_{1}\left(\Gamma^{3}, \mathbb{Z}\right)$ maps into $H_{1}(\Gamma, \mathbb{Z})$. It remains to prove that $H_{1}\left(\Gamma^{3}, \mathbb{Z}\right)$ surjects onto $H_{1}(\Gamma, \mathbb{Z})$. We use again Corollary 2.4.5, according to which any $c \in H_{1}(\Gamma, \mathbb{Z})$ has the form $c=\sum_{S \in \operatorname{Set}^{1} \Gamma} r_{S}(c) \sum_{i=1}^{\# S} e_{S, i}$, with $r_{S}(c) \in \mathbb{Z}$. Hence

$$
c=\iota\left(\sum_{S \in \operatorname{Set}^{1} \Gamma} r_{S}(c) e_{S}\right) .
$$

At this point (iii) follows from diagram (3.3) and the fact that, by Corollary 2.4.5, $e, f$ belong to the same C1-set if and only if $e_{\mid H_{1}(\Gamma, \mathbb{Z})}^{*}=f_{\mid H_{1}(\Gamma, \mathbb{Z})}^{*}$.

The implication if of part (iii) follows from the previous parts. In order to prove the other implication, we can assume that $\Gamma$ and $\Gamma^{\prime}$ are 3 -edge connected.

We claim that, as $\Gamma$ is 3-edge connected, the functionals $e^{*}$ restricted to $H_{1}(\Gamma, \mathbb{R})$ are all non zero and distinct, as $e$ varies in $E(\Gamma)$ (and the same holds for $\Gamma^{\prime}$ of course). That $e^{*}$ is nonzero follows from the fact that $E(\Gamma)_{\operatorname{sep}}$ is empty (cf. 2.4.4). Let $e \neq f$, now $\{e\}$ and $\{f\}$ are C1-sets (by 2.3.4). By Corollary 2.4.5 the restrictions of $e^{*}$ and $f^{*}$ to $H_{1}(\Gamma, \mathbb{R})$ are different. The claim is proved.

The claim means that the intersections of the hyperplanes $\left\{e^{*}=0\right\}_{e \in E(\Gamma)}$ with $H_{1}(\Gamma, \mathbb{R})$ are all proper and distinct, and similarly for $\Gamma^{\prime}$. Now, an isomorphism $\operatorname{Del}(\Gamma) \cong \operatorname{Del}\left(\Gamma^{\prime}\right)$ induces a bijection between the sets of generating hyperplanes passing through the origin; hence, by the claim, we get a bijection $E(\Gamma) \cong E\left(\Gamma^{\prime}\right)$ (which extends to an isomorphism $C_{1}(\Gamma, \mathbb{Z}) \cong C_{1}\left(\Gamma^{\prime}, \mathbb{Z}\right)$ ).

To conclude, we now use a basic fact from graph theory (see for example Oxl92, Sect. 5.1] or [Ale04, Thm. 3.11]), according to which the 0-skeleton of the hyperplane arrangement $\left\{e^{*}=n, \quad e \in E(\Gamma), n \in \mathbb{Z}\right\}$ in $H_{1}(\Gamma, \mathbb{R})$ is the lattice $H_{1}(\Gamma, \mathbb{Z})$ itself. Therefore, we deduce that the above bijection $E(\Gamma) \cong E\left(\Gamma^{\prime}\right)$ induces an isomorphism $H_{1}(\Gamma, \mathbb{Z}) \cong H_{1}\left(\Gamma^{\prime}, \mathbb{Z}\right)$, from which we conclude that $\Gamma \equiv_{\text {cyc }} \Gamma^{\prime}$.

Remark 3.2.4. A special case of Proposition 3.2.3 has been proved by Artamkin using a different language. In Art06, he associates to a graph $\Gamma$ a convex integral polytope $\Delta(\Gamma)$ in $H_{1}(\Gamma, \mathbb{R})$, called the "simple cycle polytope", and he proves that a 3-connected graph $\Gamma$ is uniquely determined by $\Delta(\Gamma)$ (see [Art06, Thm. 1]). $\Delta(\Gamma)$ turns out to be the union of the maximal dimensional Delaunay cells that have a vertex in the origin; hence knowing $\Delta(\Gamma)$ is equivalent to knowing $\operatorname{Del}(\Gamma)$. Using this observation and 2.2.6. Art06, Thm. 1] is equivalent to Proposition 3.2.3 (iii), provided that $\Gamma$ and $\Gamma^{\prime}$ are 3 -connected.

\subsection{Proof of Theorem 3.1.1; necessity.}

Proof. Assume that $\mathrm{Alb}(\Gamma) \cong \operatorname{Alb}\left(\Gamma^{\prime}\right)$. Using 3.1.3. we can assume that $\Gamma$ and $\Gamma^{\prime}$ are 2-edge connected. We fix a totally cyclic orientation on them. Since the Delaunay decomposition is completely determined by $\left(H_{1}(\Gamma, \mathbb{Z}) ;(),\right)$, i.e. by $\operatorname{Alb}(\Gamma)$ (see KS00, Sec. 2]), we have $\operatorname{Del}(\Gamma) \cong \operatorname{Del}\left(\Gamma^{\prime}\right)$. We can thus apply Proposition 3.2.3 (iii), getting that $\Gamma^{3} \equiv_{\text {cyc }} \Gamma^{\prime 3}$. Therefore, by Proposition 2.3.9)(iii) there is a natural bijection,

$$
\operatorname{Set}^{1} \Gamma \stackrel{\beta}{\longrightarrow} \operatorname{Set}^{1} \Gamma^{\prime} ; \quad S \mapsto S^{\prime}:=\beta(S) .
$$


To prove the theorem it suffices to show that $\beta$ preserves the cardinalities. In fact by Proposition 2.3.9(iii), this implies that $\Gamma \equiv_{\text {cyc }} \Gamma^{\prime}$.

First, note that by hypothesis there is an isomorphism, denoted

$$
H_{1}(\Gamma, \mathbb{Z}) \stackrel{\cong}{\longrightarrow} H_{1}\left(\Gamma^{\prime}, \mathbb{Z}\right) ; \quad c \mapsto c^{\prime}
$$

such that $\left(c_{1}, c_{2}\right)=\left(c_{1}^{\prime}, c_{2}^{\prime}\right)$ for all $c_{i} \in H_{1}(\Gamma, \mathbb{Z})$. Pick $c \in H_{1}(\Gamma, \mathbb{Z})$; by Corollary 2.4.5 we can write $c=\sum_{S \in \operatorname{Set}^{1} \Gamma} r_{S}(c) \sum_{i=1}^{\# S} e_{S, i}$, with $r_{S}(c) \in \mathbb{Z}$; hence we can define (consistently with 2.3 .3 ) the set

$$
\operatorname{Set}_{c}^{1} \Gamma:=\left\{S \in \operatorname{Set}^{1} \Gamma: r_{S}(c) \neq 0\right\} .
$$

We claim that for every $S \in \operatorname{Set}^{1} \Gamma$ and every $c \in H_{1}(\Gamma, \mathbb{Z})$ we have

$$
r_{S^{\prime}}\left(c^{\prime}\right)=u(S) r_{S}(c), \quad u(S):= \pm 1
$$

in particular,

$$
S \in \operatorname{Set}_{c}^{1} \Gamma \Leftrightarrow S^{\prime} \in \operatorname{Set}_{c^{\prime}}^{1} \Gamma^{\prime} .
$$

To prove the claim, consider the affine function $f_{S}^{n}: C_{1}(\Gamma, \mathbb{Z}) \rightarrow \mathbb{Z}$ defined as

$$
f_{S}^{n}:=e_{S}^{*}-n, \quad n \in \mathbb{Z} .
$$

By what we said before we have

$$
r_{S}(c)=n \Leftrightarrow c \in \operatorname{ker} f_{S}^{n} .
$$

Observe that the bijections (3.5) and (3.4) are compatible with one another. In other words, for every $c \in H_{1}(\Gamma, \mathbb{Z})$, the set $\operatorname{Set}_{c}^{1} \Gamma$ is mapped to $\operatorname{Set}_{c^{\prime}}^{1} \Gamma^{\prime}$ by $\beta$. Therefore the isomorphism between $\operatorname{Alb}(\Gamma)$ and $\operatorname{Alb}\left(\Gamma^{\prime}\right)$ induces a bijection between the hyperplanes generating $\operatorname{Del}(\Gamma)$ and those generating $\operatorname{Del}\left(\Gamma^{\prime}\right)$ such that $f_{S}^{n}$ is mapped either to $f_{S^{\prime}}^{n}$ or to $f_{S^{\prime}}^{-n}$ (see Remark 3.2.2). So, the claim is proved.

To ease the notation, in the sequel for any $S \in \operatorname{Set}^{1} \Gamma$ we denote

$$
e(S):=\sum_{i=1}^{\# S} e_{S, i}
$$

Moreover, if $S \in \operatorname{Set}_{c}^{1} \Gamma$ for some $c$, we denote

$$
\lambda(c-S):=\sum_{T \in \operatorname{Set}_{c}^{1} \Gamma \backslash\{S\}} \# T .
$$

Observe that for any cycle $\Delta \subset \Gamma$ of length $\lambda$ and any $c:=\sum_{e \in E(\Delta)} \pm e \in H_{1}(\Gamma, \mathbb{Z})$ (such a $c$ exists for a suitable choice of signs), we have $\operatorname{Set}_{\Delta}^{1} \Gamma=\operatorname{Set}_{c}^{1} \Gamma$ and

$$
\lambda=\|c\|^{2}=\sum_{S \in \operatorname{Set}_{\Delta}^{1} \Gamma} \# S=\# S+\lambda(c-S)
$$

for any $S \in \operatorname{Set}_{\Delta}^{1} \Gamma$.

We shall now prove that the map (3.4) preserves cardinalities. By contradiction, suppose there exists $S \in \operatorname{Set}^{1} \Gamma$ such that

$$
\# S>\# S^{\prime}
$$

By Lemma 3.3.1, we can find two cycles $\Delta_{1}$ and $\Delta_{2}$ of $\Gamma$ such that $S=E\left(\Delta_{1}\right) \cap$ $E\left(\Delta_{2}\right)$. For $i=1,2$, there exists an element $c_{i} \in H_{1}(\Gamma, \mathbb{Z})$ given by the formula

$$
c_{i}=e(S)+\sum_{T \in \operatorname{Set}_{c_{i}}^{1} \Gamma \backslash\{S\}} \pm e(T)
$$

(by Corollary 2.4.5). The sign before $e(T)$ will play no role, so we can ignore it. 
Suppose to fix ideas that $u(S)=1$ in (3.6). The case $u(S)=-1$ is treated in a trivially analogous way (we omit the details). By (3.6), we have

$$
c_{i}^{\prime}=e\left(S^{\prime}\right)+\sum_{T \in \operatorname{Set}_{c_{i}}^{1} \Gamma \backslash\{S\}} \pm e\left(T^{\prime}\right)
$$

(recall that $T$ determines $T^{\prime}$ uniquely). Therefore, as $\left\|c_{i}\right\|^{2}=\left(c_{i}, c_{i}\right)=\left(c_{i}^{\prime}, c_{i}^{\prime}\right)=$ $\left\|c_{i}^{\prime}\right\|^{2}$, using notation (3.8)

$$
\left\|c_{i}\right\|^{2}=\# S+\lambda\left(c_{i}-S\right)=\left\|c_{i}^{\prime}\right\|^{2}=\# S^{\prime}+\lambda\left(c_{i}^{\prime}-S^{\prime}\right) .
$$

By (3.9) we get for $i=1,2$

$$
\lambda\left(c_{i}-S\right)<\lambda\left(c_{i}^{\prime}-S^{\prime}\right) .
$$

Now, $c_{1}-c_{2}$ lies, of course, in $H_{1}(\Gamma, \mathbb{Z})$; we have

$$
c_{1}-c_{2}=\sum_{T \in \operatorname{Set}_{c_{1}}^{1} \Gamma \backslash\{S\}} \pm e(T)-\sum_{U \in \operatorname{Set}_{c_{2}}^{1} \Gamma \backslash\{S\}} \pm e(U) .
$$

Since $\operatorname{Set}_{c_{1}}^{1} \Gamma \cap \operatorname{Set}_{c_{2}}^{1} \Gamma=\{S\}$, we have

$$
\left\|c_{1}-c_{2}\right\|^{2}=\lambda\left(c_{1}-S\right)+\lambda\left(c_{2}-S\right) .
$$

Arguing in the same way for $c_{1}^{\prime}-c_{2}^{\prime}$, we get

$$
\left\|c_{1}^{\prime}-c_{2}^{\prime}\right\|^{2}=\lambda\left(c_{1}^{\prime}-S^{\prime}\right)+\lambda\left(c_{2}^{\prime}-S^{\prime}\right) .
$$

Therefore, using (3.12), (3.10) and (3.11)

$$
\left\|c_{1}^{\prime}-c_{2}^{\prime}\right\|^{2}=\lambda\left(c_{1}^{\prime}-S^{\prime}\right)+\lambda\left(c_{2}^{\prime}-S^{\prime}\right)>\lambda\left(c_{1}-S\right)+\lambda\left(c_{2}-S\right)=\left\|c_{1}-c_{2}\right\|^{2}
$$

which contradicts the fact that the isomorphism (3.5) preserves the scalar products.

In the proof we applied the next Lemma, which will be used again later on.

Lemma 3.3.1. Let $S \in \operatorname{Set}^{1} \Gamma$. For every cycle $\Delta \subset \Gamma$ such that $S \subset E(\Delta)$ there exists a cycle $\hat{\Delta} \subset \Gamma$ such that $S=E(\Delta) \cap E(\hat{\Delta})$.

Proof. It is clear that it suffices to assume $\Gamma$ free from separating edges. We begin by reducing to the case $\# S=1$. Choose an edge $e \in S$ and consider the map $\Gamma \rightarrow \bar{\Gamma}$ contracting all edges of $S$ but $e$. Then $\sigma$ induces a bijection between the cycles of $\Gamma$ and those of $\bar{\Gamma}$, and it is clear that if the statement holds on $\bar{\Gamma}$ it also holds on $\Gamma$.

So, let $S=\{e\}$ and let $\Delta$ be a cycle containing $e$. We shall exhibit an iterated procedure which yields, at its $i$-th step, a cycle $\Delta_{i}$ containing $e$ and such that $\# E(\Delta) \cap E\left(\Delta_{i}\right)$ decreases at each step. Set $\Delta_{1}=\Delta$ and $S_{1}:=S=\{e\}$; if $\Delta$ has length 1 we take $\hat{\Delta}=\Delta$ and we are done. So, suppose $\# E(\Delta) \geq 2$; we can decompose $E(\Delta)$ as a disjoint union of C1-sets $E(\Delta)=\{e\} \cup S_{2} \cup \ldots \cup S_{h}$, with $S_{i} \in \operatorname{Set}^{1} \Gamma$ (cf. Remark 2.3.3). For the second step consider $\Gamma_{2}:=\Gamma \backslash S_{2}$; then $\Gamma_{2}$ has no separating edges, therefore there exists a cycle $\Delta_{2} \subset \Gamma_{2}$ containing $e$. Obviously $\Delta_{2}$ does not contain $S_{2}$, hence $\# E(\Delta) \cap E\left(\Delta_{2}\right)<\# E(\Delta) \cap E\left(\Delta_{1}\right)$. If $\Delta_{2}$ does not contain any other edge of $\Delta$ we take $\Delta_{2}=\hat{\Delta}$ and we are done. Otherwise we repeat the process within $\Gamma_{2}$. Namely, we have $E\left(\Delta_{2}\right)=\{e\} \cup S_{2}^{2} \cup \ldots \cup S_{h}^{2}$, with $S_{i}^{2} \in \operatorname{Set}^{1} \Gamma_{2}$, set $\Gamma_{3}:=\Gamma_{2} \backslash S_{2}^{2}$. There exists a cycle $\Delta_{3} \subset \Gamma_{3}$ containing $e$, and it is clear that $\# E(\Delta) \cap E\left(\Delta_{3}\right)<\# E(\Delta) \cap E\left(\Delta_{2}\right)$.

Obviously this process must terminate after, say $m$, steps, when we necessarily have $E(\Delta) \cap E\left(\Delta_{m}\right)=\{e\}$. 


\section{TORELLI THEOREM FOR METRIC GRAPHS AND TROPICAL CURVES}

In this section we apply the methods and results of the previous part to study the Torelli problem for tropical curves. We refer to Mik06, or to [MZ07, for details about the theory of tropical curves and their Jacobians.

4.1. Tropical curves, metric graphs and associated tori. Let $C$ be a compact tropical curve; $C$ is endowed with a Jacobian variety, $\operatorname{Jac}(C)$, which is a principally polarized tropical Abelian variety (see [MZ07, Sec. 5] and Mik06, Sect $5.2])$; we shall denote $\left(\operatorname{Jac}(C), \Theta_{C}\right)$ the principally polarized Jacobian of $C$, where $\Theta_{C}$ denotes the principal polarization (see Remark 4.1.5 below). Observe that two tropically equivalent curves have isomorphic Jacobians. As we stated in the introduction, we want to study the following

Problem. For which compact tropical curves $C$ and $C^{\prime}$ there is an isomorphism $\left(\operatorname{Jac}(C), \Theta_{C}\right) \cong\left(\operatorname{Jac}\left(C^{\prime}\right), \Theta_{C^{\prime}}\right)$ ?

We will answer this question in Theorem 4.1.9.

As we already mentioned, the connection with the earlier sections of this paper comes from a result of G. Mikhalkin and I. Zharkov, establishing that tropical curves are closely related to metric graphs.

Definition 4.1.1. A metric graph $(\Gamma, l)$ is a finite graph $\Gamma$ endowed with a function $l: E(\Gamma) \rightarrow \mathbb{R}_{>0}$ called the length function.

Remark 4.1.2. Our definition of metric graph coincides with that of [MZ07] only if the graph has valence at least 2. The difference occurs in the length function, whereas the graph is the same. More precisely, the definition of length function used in MZ07 differs from ours, as it assigns the value $+\infty$ to every edge adjacent to a vertex of valence 1 ; such edges are called leaves. With this definition, metric graphs are in bijection with tropical curves.

To avoid trivial cases, we shall always assume that our tropical curves have genus at least 2. Under this assumption, by [MZ07, Prop. 3.6], there is a one to one correspondence between tropical equivalence classes of compact tropical curves and metric graphs with valence at least 3 (i.e such that every vertex has at least three incident edges).

Therefore, from now on, we identify compact tropical curves, up to tropical equivalence, with metric graphs of valence at least 3 .

Remark 4.1.3. Since to every compact tropical curve $C$ we associate a unique finite graph $\Gamma$, we will use for $C$ the graph theoretic terminology. In particular, we shall say that $C$ is $k$-connected if so is $\Gamma$.

Given a metric graph $(\Gamma, l)$, we define the scalar product $(,)_{l}$ on $C_{1}(\Gamma, \mathbb{R})$ as follows

$$
\left(e, e^{\prime}\right)_{l}= \begin{cases}l(e) & \text { if } e=e^{\prime}, \\ 0 & \text { otherwise. }\end{cases}
$$

In analogy with Definitions 2.1.1, 2.2.3 and 2.3.6 we shall define the Albanese torus, the cyclic equivalence, and the 3-edge connectivization for metric graphs.

Definition 4.1.4. The Albanese torus $\operatorname{Alb}(\Gamma, l)$ of the metric graph $(\Gamma, l)$ is

$$
\operatorname{Alb}(\Gamma, l):=\left(H_{1}(\Gamma, \mathbb{R}) / H_{1}(\Gamma, \mathbb{Z}) ;(,)_{l}\right)
$$

(with the flat metric derived from the scalar product $\left.(,)_{l}\right)$.

Remark 4.1.5. By MZ07, Sect. 6.1 p. 218] we can naturally identify $\left(\operatorname{Jac}(C), \Theta_{C}\right)$ with the Albanese torus $\operatorname{Alb}(\Gamma, l)$. 
Definition 4.1.6. Let $(\Gamma, l)$ and $\left(\Gamma^{\prime}, l^{\prime}\right)$ be two metric graphs. We say that $(\Gamma, l)$ and $\left(\Gamma^{\prime}, l^{\prime}\right)$ are cyclically equivalent. and we write $(\Gamma, l) \equiv_{\text {cyc }}\left(\Gamma^{\prime}, l^{\prime}\right)$, if there exists a cyclic bijection $\epsilon: E(\Gamma) \rightarrow E\left(\Gamma^{\prime}\right)$ such that $l(e)=l^{\prime}(\epsilon(e))$ for all $e \in E(\Gamma)$. The cyclic equivalence class of $(\Gamma, l)$ will be denoted by $[(\Gamma, l)]_{\text {cyc }}$.

Definition 4.1.7. A 3-edge connectivization of a metric graph $(\Gamma, l)$ is a metric graph $\left(\Gamma^{3}, l^{3}\right)$, where $\Gamma^{3}$ is a 3 -edge connectivization of $\Gamma$, and $l^{3}$ is the length function defined as follows,

$$
l^{3}\left(e_{S}\right)=\sum_{e \in \psi^{-1}\left(e_{S}\right)} l(e)=\sum_{e \in S} l(e)
$$

where, with the notation of (2.4), $\psi: \operatorname{Set}^{1} \Gamma \rightarrow E\left(\Gamma^{3}\right)$ is the natural bijection mapping $S$ to $e_{S}$.

Remark 4.1.8. Using lemma 2.3.8(iii) we see that all the 3-edge connectivizations of a metric graph $(\Gamma, l)$ are cyclically equivalent.

Observe also that $\left[\left(\Gamma^{3}, l^{3}\right)\right]_{\mathrm{cyc}}$ is completely independent of the separating edges of $\Gamma$, and on the value that $l$ takes on them. Therefore, $\left(\Gamma^{3}, l^{3}\right)$ is well defined also if $l$ takes value $+\infty$ on the leaves of $\Gamma$. This enables us to define $\left[\left(\Gamma^{3}, l^{3}\right)\right]_{\mathrm{cyc}}$ for a graph $(\Gamma, l)$, metric in the sense of [MZ07, associated to a tropical curve $C$ (see Remark 4.1.2).

Consistently with Remark 2.3.10 we call $\left[\left(\Gamma^{3}, l^{3}\right)\right]_{\text {cyc }}$ the 3-edge connected class of $C$. With this terminology, we state the main result of this section:

Theorem 4.1.9. Let $C$ and $C^{\prime}$ be compact tropical curves. Then $\left(\operatorname{Jac} C, \Theta_{C}\right) \cong$ (Jac $C^{\prime}, \Theta_{C^{\prime}}$ ) if and only if $C$ and $C^{\prime}$ have the same 3-edge connected class.

Suppose that $C$ is 3-connected. Then $\left(\mathrm{Jac} C, \Theta_{C}\right) \cong\left(\mathrm{Jac} C^{\prime}, \Theta_{C^{\prime}}\right)$ if and only if $C$ and $C^{\prime}$ are tropically equivalent.

Proof. The first statement is a straightforward consequence of the next Theorem 4.1.10.

We let $\Gamma$ and $\Gamma^{\prime}$ be the metric graphs associated to $C$ and $C^{\prime}$ respectively. Suppose now that $C$ is 3-connected. This means (cf. 4.1.3) that the associated graph is 3 -connected (and hence 3 -edge connected). By the previous part $\Gamma=\Gamma^{3} \cong$ $\Gamma^{\prime 3}$. Recall that, by convention (cf. Remark 4.1.2), the graph $\Gamma^{\prime}$ has valence at least 3. To finish the proof it suffices to show that the map $\sigma: \Gamma^{\prime} \rightarrow \Gamma^{\prime 3}$ is the identity map; to do that we will use the fact that $\Gamma^{\prime 3}$ is 3 -connected, as $\Gamma$ is.

Suppose $\sigma$ contracts a separating edge $e$ of $\Gamma^{\prime}$; observe that the two vertices adjacent to $e$ are both separating vertices for $\Gamma^{\prime}$, because $\Gamma^{\prime}$ has no vertices of valence 1 . But then $\sigma(e)$ would be a separating vertex of $\Gamma^{\prime 3}$, which is impossible.

If $\sigma$ contracts one edge of a separating pair, arguing in a similar way we obtain that $\Gamma^{\prime}$ has a separating pair of vertices which is mapped by $\sigma$ to a separating pair of vertices of $\Gamma^{\prime 3}$, which is impossible. Therefore $\sigma$ is the identity and we are done.

Theorem 4.1.10. Let $(\Gamma, l)$ and $\left(\Gamma^{\prime}, l^{\prime}\right)$ be two metric graphs. Then $\operatorname{Alb}(\Gamma, l) \cong$ $\operatorname{Alb}\left(\Gamma^{\prime}, l^{\prime}\right)$ if and only if $\left[\left(\Gamma^{3}, l^{3}\right)\right]_{\mathrm{cyc}}=\left[\left(\Gamma^{\prime 3}, l^{\prime 3}\right)\right]_{\mathrm{cyc}}$.

4.2. Proof of the Torelli theorem for metric graphs. The proof of Theorem 4.1.10 follows the same steps as the proof of Theorem 3.1.1 The "if" part follows easily from the following

Proposition 4.2.1. Let $(\Gamma, l)$ be a metric graph.

(i) $\operatorname{Alb}(\Gamma, l)$ depends only on $[(\Gamma, l)]_{\text {cyc }}$.

(ii) $\operatorname{Alb}(\Gamma, l) \cong \operatorname{Alb}\left(\Gamma^{3}, l^{3}\right)$ for any 3 -edge connectivization of $(\Gamma, l)$. 
Proof. Part (ii) follows from the fact that $\left(H_{1}(\Gamma, \mathbb{Z}) ;(,)_{l}\right)$ is defined entirely in terms of the inclusion $H_{1}(\Gamma, \mathbb{Z}) \subset C_{1}(\Gamma, \mathbb{Z})$ and of the values of $(,)_{l}$ on the orthogonal basis $E(\Gamma)$ of $C_{1}(\Gamma, \mathbb{Z})$, all of which is clearly invariant by cyclic equivalence.

To prove part (iii) we use the proof of Proposition 3.2.3(iii), to which we now refer for the notation.

Consider the diagram (3.3). The point is that the inclusion $\iota$ is compatible with the scalar product $(,)_{l}$ on the right and the scalar product $(,)_{l^{3}}$ on the left. More precisely, for every edge $e_{S}$ of $\Gamma^{3}$ (so that $S \in \operatorname{Set}^{1} \Gamma$ ) we have (by definition of $l^{3}$ )

$$
\left(e_{S}, e_{S}\right)_{l^{3}}=l^{3}\left(e_{S}\right)=\sum_{e \in S} l(e)=\left(\sum_{i=1}^{\# S} e_{S, i}, \sum_{i=1}^{\# S} e_{S, i}\right)_{l}=\left(\iota\left(e_{S}\right), \iota\left(e_{S}\right)\right)_{l} .
$$

On the other hand if $T \in \operatorname{Set}^{1} \Gamma$ with $T \neq S$ we have $0=\left(e_{S}, e_{T}\right)_{l^{3}}=\left(\iota\left(e_{S}\right), \iota\left(e_{T}\right)\right)_{l}$ (since $S \cap T=\emptyset$ ).

Therefore (ii) is proved, and with it the sufficiency part of Theorem 4.1.10.

To prove the opposite implication of Theorem 4.1.10, we need the following

Definition 4.2.2. The Delaunay decomposition $\operatorname{Del}(\Gamma, l)$ associated to the metric graph $(\Gamma, l)$ is the Delaunay decomposition (cf. Definition 3.2.1) associated to the scalar product $(,)_{l}$ on $H_{1}(\Gamma, \mathbb{R})$ with respect to the lattice $H_{1}(\Gamma, \mathbb{Z})$.

Lemma 4.2.3. Let $(\Gamma, l)$ be a metric graph. Then

(i) $\operatorname{Del}(\Gamma, l)$ is determined by $\operatorname{Alb}(\Gamma, l)$.

(ii) $\operatorname{Del}(\Gamma, l)=\operatorname{Del}(\Gamma)$.

Proof. Clearly, $\operatorname{Del}(\Gamma, l)$ is determined by the lattice $H_{1}(\Gamma, \mathbb{Z}) \subset H_{1}(\Gamma, \mathbb{R})$ and the scalar product $(,)_{l}$, and therefore by $\operatorname{Alb}(\Gamma, l)$. This shows part (ii).

Part (iii) follows from a well-known Theorem of Mumford, Nam76, Thm 18.2].

Proof of Theorem 4.1.10: necessity. Suppose that $\operatorname{Alb}(\Gamma, l) \cong \operatorname{Alb}\left(\Gamma^{\prime}, l^{\prime}\right)$. By Lemma 4.2.3. $(\Gamma, l)$ and $\left(\Gamma^{\prime}, l^{\prime}\right)$ have the same Delaunay decompositions and $\operatorname{Del}(\Gamma)=$ $\operatorname{Del}\left(\Gamma^{\prime}\right)$. We can assume that $\Gamma$ and $\Gamma^{\prime}$ are 3 -edge connected. By Proposition 3.2.3(iii), we have $\Gamma \equiv_{\text {cyc }} \Gamma^{\prime}$. Denote

$$
E(\Gamma) \stackrel{\epsilon}{\longrightarrow} E\left(\Gamma^{\prime}\right) ; \quad e \mapsto e^{\prime}:=\epsilon(e)
$$

a cyclic bijection. It remains to prove that $l(e)=l^{\prime}\left(e^{\prime}\right)$ for every $e \in E(\Gamma)$. We will proceed in strict analogy with the proof of the necessity of Theorem 3.1.1.

First, note that there is an isomorphism, denoted

$$
H_{1}(\Gamma, \mathbb{Z}) \stackrel{\cong}{\longrightarrow} H_{1}\left(\Gamma^{\prime}, \mathbb{Z}\right) ; \quad c \mapsto c^{\prime}
$$

such that $\left(c_{1}, c_{2}\right)_{l}=\left(c_{1}^{\prime}, c_{2}^{\prime}\right)_{l^{\prime}}$ for all $c_{i} \in H_{1}(\Gamma, \mathbb{Z})$. Pick $c \in H_{1}(\Gamma, \mathbb{Z})$ and write $c=$ $\sum_{e \in E(\Gamma)} r_{e}(c) e$, with $r_{e}(c) \in \mathbb{Z}$; similarly $c^{\prime}=\sum_{e^{\prime} \in E\left(\Gamma^{\prime}\right)} r_{e^{\prime}}\left(c^{\prime}\right) e^{\prime}$ with $r_{e^{\prime}}\left(c^{\prime}\right) \in \mathbb{Z}$. We claim that for every $e \in E(\Gamma)$ and every $c \in H_{1}(\Gamma, \mathbb{Z})$ we have

$$
r_{e^{\prime}}\left(c^{\prime}\right)=u(e) r_{e}(c), \quad u(e):= \pm 1 .
$$

To prove the claim, notice that $r_{e}(c)=n \Leftrightarrow e^{*}(c)=n$. On the other hand, the isomorphism between $\operatorname{Del}(\Gamma)$ and $\operatorname{Del}\left(\Gamma^{\prime}\right)$ maps the hyperplane of equation $e^{*}=n$ either to $e^{\prime *}=n$ or to $e^{\prime *}=-n$. So, the claim is proved. Now define $E_{c}(\Gamma):=\left\{e \in E(\Gamma): r_{e}(c) \neq 0\right\}$. For any $c \in H_{1}(\Gamma, \mathbb{Z})$ and $e \in E_{c}(\Gamma)$ we shall denote

$$
\lambda(c-e):=\sum_{f \in E_{c}(\Gamma) \backslash\{e\}} l(f) .
$$


We can now prove that the map (4.1) preserves the lengths, i.e. that $l(e)=l^{\prime}\left(e^{\prime}\right)$ for every $e \in E(\Gamma)$. By contradiction, suppose there exists an edge $e$ of $\Gamma$ such that

$$
l(e)>l^{\prime}\left(e^{\prime}\right) .
$$

By Lemma 3.3.1, there exist two cycles $\Delta_{1}$ and $\Delta_{2}$ of $\Gamma$ such that $\{e\}=E\left(\Delta_{1}\right) \cap$ $E\left(\Delta_{2}\right)$. As in the proof of Theorem 3.1.1, consider $c_{1}$ and $c_{2}$ in $H_{1}(\Gamma, \mathbb{Z})$ associated to the above two cycles (so that $E_{c_{i}}(\Gamma)=\operatorname{Set}_{\Delta_{i}}^{1} \Gamma$ for $i=1,2$ ):

$$
c_{i}=e+\sum_{f \in E_{c_{i}}(\Gamma) \backslash\{e\}} \pm f .
$$

The sign before $f$ will play no role, hence we are free to ignore it. Suppose that $u(e)=1$ (the case $u(e)=-1$ is treated similarly). By (4.3) we have

$$
c_{i}^{\prime}=e^{\prime}+\sum_{f \in E_{c_{i}}(\Gamma) \backslash\{e\}} \pm f^{\prime} .
$$

Therefore, as $\left\|c_{i}\right\|^{2}:=\left(c_{i}, c_{i}\right)_{l}=\left(c_{i}^{\prime}, c_{i}^{\prime}\right)_{l^{\prime}}=:\left\|c_{i}^{\prime}\right\|^{2}$, using notation (4.4) we have

$$
\left\|c_{i}\right\|^{2}=l(e)+\lambda\left(c_{i}-e\right)=\left\|c_{i}^{\prime}\right\|^{2}=l\left(e^{\prime}\right)+\lambda\left(c_{i}^{\prime}-e^{\prime}\right) .
$$

By (4.5) we get

$$
\lambda\left(c_{i}-e\right)<\lambda\left(c_{i}^{\prime}-e^{\prime}\right)
$$

Now consider $c_{1}-c_{2} \in H_{1}(\Gamma, \mathbb{Z})$. We have

$$
c_{1}-c_{2}=\sum_{f \in E_{c_{1}}(\Gamma) \backslash\{e\}} \pm f-\sum_{g \in E_{c_{2}}(\Gamma) \backslash\{e\}} \pm g .
$$

Since $E_{c_{1}}(\Gamma) \cap E_{c_{2}}(\Gamma)=\{e\}$, we have $\left\|c_{1}-c_{2}\right\|^{2}=\lambda\left(c_{1}-e\right)+\lambda\left(c_{2}-e\right)$. Arguing similarly for $c_{1}^{\prime}-c_{2}^{\prime}$, we get $\left\|c_{1}^{\prime}-c_{2}^{\prime}\right\|^{2}=\lambda\left(c_{1}^{\prime}-e^{\prime}\right)+\lambda\left(c_{2}^{\prime}-e^{\prime}\right)$. Hence, by (4.6)

$$
\left\|c_{1}^{\prime}-c_{2}^{\prime}\right\|^{2}=\lambda\left(c_{1}^{\prime}-e^{\prime}\right)+\lambda\left(c_{2}^{\prime}-e^{\prime}\right)>\lambda\left(c_{1}-e\right)+\lambda\left(c_{2}-e\right)=\left\|c_{1}-c_{2}\right\|^{2},
$$

contradicting the fact that (4.2) preserves the scalar products.

\section{FURTHER CHARACTERIZATIONS OF GRAPHS}

The Torelli theorems proved in the previous sections are based on the notion of 3 -edge connected class, $\left[\Gamma^{3}\right]_{\mathrm{cyc}}$, of a graph $\Gamma$. The aim of this section, whose main result is Theorem 5.3 .2 , is to give some other characterizations of $\left[\Gamma^{3}\right]_{\text {cyc }}$.

\subsection{The poset $\mathcal{S} \mathcal{P}_{\Gamma}$.}

Definition 5.1.1. Let $\Gamma$ be a graph. The poset $\mathcal{S} \mathcal{P}_{\Gamma}$ is the set of all the subsets $S \subset E(\Gamma)$ such that the subgraph $\Gamma \backslash S$ is free from separating edges, endowed with the following partial order:

$$
S \geq T \Longleftrightarrow S \subseteq T \text {. }
$$

Remark 5.1.2. It is clear that for every $S \in \mathcal{S P}_{\Gamma}$ we have $E(\Gamma)_{\text {sep }} \subset S$. Therefore any map $\sigma: \Gamma \rightarrow \bar{\Gamma}$ contracting some separating edges of $\Gamma$ induces a bijection of posets $\mathcal{S P}_{\Gamma} \longrightarrow \mathcal{S} \mathcal{P}_{\bar{\Gamma}}$.

We will use some notions and facts from graph theory.

Definition 5.1.3. Oxl92, Sect. 2.3] The cographic matroid $M^{*}(\Gamma)$ of $\Gamma$ is the matroid of collections of linearly independent vectors among the collections of vectors $\left\{e^{*}: e \in E(\Gamma)\right\}$ of $H_{1}(\Gamma, \mathbb{R})^{*}$.

Remark 5.1.4. It is well known that the cographic matroid $M(\Gamma)$ is independent of the choice of the orientation of $\Gamma$ used to define $H_{1}(\Gamma, \mathbb{Z}) \subset C_{1}(\Gamma, \mathbb{Z})$.

Theorem 5.1.5. Oxl92, Sect. 5.3] $M^{*}(\Gamma) \cong M^{*}\left(\Gamma^{\prime}\right)$ if and only if $\Gamma \equiv_{\text {cyc }} \Gamma^{\prime}$. 
We are going to show that the poset $\mathcal{S} \mathcal{P}_{\Gamma}$ is determined by $M^{*}(\Gamma)$. Before doing that we recall the notion of a flat of the matroid $M^{*}(\Gamma)$ (see for example Oxl92, Sec. 1.7]). First, for any $S=\left\{e_{S, 1}, \ldots, e_{S, \# S}\right\} \subset E(\Gamma)$ we denote by

$$
\left\langle S^{*}\right\rangle=\operatorname{span}\left(e_{S, 1}^{*}, \ldots, e_{S, \# S}^{*}\right) \subset H_{1}(\Gamma, \mathbb{Z})^{*} .
$$

We say that $S$ is a flat of $M^{*}(\Gamma)$ if for every $e \in E(\Gamma) \backslash S$ we have

$$
\operatorname{dim}\left\langle S^{*}\right\rangle<\operatorname{dim} \operatorname{span}\left(S^{*}, e^{*}\right)=\operatorname{dim} \operatorname{span}\left(e_{S, 1}^{*}, \ldots, e_{S, \# S}^{*}, e^{*}\right) .
$$

Lemma 5.1.6. $\mathcal{S} \mathcal{P}_{\Gamma}$ is the set of flats of the matroid $M^{*}(\Gamma)$.

Proof. Given any subset $T \subset E(\Gamma)$, its closure $\operatorname{cl}(T)$ is defined as the subset of $E(\Gamma)$ formed by all the $e \in E(\Gamma)$ such that $e^{*} \in \operatorname{span}_{f \in T}\left(f^{*}\right)$. It is clear that $T \subset E(\Gamma)$ is a flat if and only if $T=\operatorname{cl}(T)$.

We have the following commutative diagram

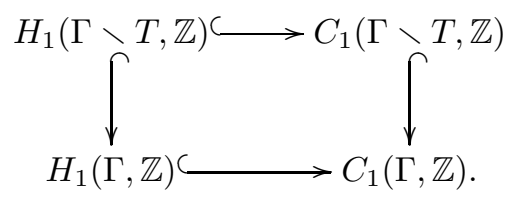

The left vertical injective map induces a surjective map $H_{1}(\Gamma, \mathbb{Z})^{*} \rightarrow H_{1}(\Gamma \backslash T, \mathbb{Z})^{*}$ whose kernel is equal to $\operatorname{span}_{f \in T}\left(f^{*}\right)$. Therefore $e \in \operatorname{cl}(T)$ if and only the image, $\left[e^{*}\right]$, of $e^{*}$ under the above surjection is zero. If $e \notin T$ then $\left[e^{*}\right]=0$ if and only if $e$ does not belong to any cycle of $\Gamma \backslash T$, if and only if $e$ is a separating edge of $\Gamma \backslash T$. This shows that $T=\operatorname{cl}(T)$ if and only $\Gamma \backslash T$ does not have separating edges, in other words $T$ is a flat if and only $T \in \mathcal{S} \mathcal{P}_{\Gamma}$.

Remark 5.1.7. It is easy to see, from the above definitions, that the poset of flats of $M^{*}(\Gamma)$ is isomorphic to the poset of intersections of the arrangement of hyperplanes $\left\{e^{*}=0\right\}_{e \in E(\Gamma) \backslash E(\Gamma)_{\mathrm{sep}}}$.

Recall that a poset $(P, \leq)$ is called graded if it is has a monotone function $\rho$ : $P \rightarrow \mathbb{N}$, called the rank function, such that if $x$ covers $y$ (i.e. $y \lesseqgtr x$ and there does not exist a $z$ such that $y \leq z \leq x)$ then $\rho(x)=\rho(y)+1$. If our poset has a minimum element $\underline{0}$, we say that it is bounded from below. If this is the case $(P, \leq)$ is graded if and only if for every element $x \in P$ all the maximal chains from $\underline{0}$ to $x$ have the same length. We can define a rank function $\rho: P \rightarrow \mathbb{N}$ by setting $\rho(x)$ equal to the length of any chain from $\underline{0}$ to $x$. This is the unique rank function on $(P, \leq)$ such that $\rho(\underline{0})=0$ and we call it the normalized rank function.

Corollary 5.1.8. The poset $\mathcal{S P}_{\Gamma}$ is a graded poset with minimum element equal to $E(\Gamma)$ and normalized rank function given by $S \mapsto b_{1}(\Gamma \backslash S)$.

Proof. (It is well-known, see Oxl92, Thm. 1.7.5], that the poset of flats of a matroid is a geometric lattice; hence in particular a graded poset.) The minimum element is clearly $E(\Gamma)$ and the length of a chain in $\mathcal{S P}_{\Gamma}$ from $E(\Gamma)$ to $S$ is exactly equal to the number of independent cycles in $\Gamma \backslash S$, that is to $b_{1}(\Gamma \backslash S)$.

Remark 5.1.9. We like to think of the number $b_{1}(\Gamma \backslash S)$ as the codimension of the set $S \in \mathcal{S P}_{\Gamma}$. If $E(\Gamma)_{\text {sep }}=\emptyset$ (which is a harmless assumption, by remark 5.1.2), then $\operatorname{Set}^{1} \Gamma \subset \mathcal{S} \mathcal{P}_{\Gamma}$, and we have that $S$ has codimension 1 if and only if $S$ is a C1-set. (cf. 2.3.1).

Lemma 5.1.10. Let $\Gamma$ and $\Gamma^{\prime}$ two graphs. For any choice of $\Gamma^{3}$ and $\Gamma^{\prime 3}$ we have:

(i) $\mathcal{S} \mathcal{P}_{\Gamma} \cong \mathcal{S} \mathcal{P}_{\Gamma^{3}}$ (as posets).

(ii) $\mathcal{S} \mathcal{P}_{\Gamma} \cong \mathcal{S} \mathcal{P}_{\Gamma^{\prime}}$ if and only if $\Gamma^{3} \equiv_{\text {cyc }} \Gamma^{\prime 3}$. 
Proof. It is well known that the poset of flats of a matroid $M$ depends on, and completely determines, any simple matroid $\widetilde{M}$ (see below) associated to $M$ (see Oxl92, Sec. 1.7]). Therefore, using Theorem 5.1.5, we will be done if we show that $\widetilde{M^{*}(\Gamma)}=M^{*}\left(\Gamma^{3}\right)$ for any choice of $\Gamma^{3}$ of $\Gamma$. Since the cographic matroid does not depend on the choice of the orientation (cf. Remark 5.1.4), we can fix an orientation on $\Gamma$ inducing a totally cyclic orientation on $\Gamma \backslash E(\Gamma)_{\text {sep }}$, and we let $\Gamma^{3}$ have the orientation induced by that of $\Gamma$.

Recall (see loc. cit.) that a simple matroid $\widetilde{M^{*}(\Gamma)}$ is obtained from $M^{*}(\Gamma)$ by deleting the zero vectors and, for each parallel (i.e. proportional) class of vectors, deleting all but one of the vectors. We know $e^{*} \in H_{1}(\Gamma, \mathbb{R})^{*}$ is zero if and only $e \in E(\Gamma)_{\text {sep }}$ (see 2.4.4). On the other hand, Corollary 2.4.5)(2) yields that $e_{1}^{*}$ and $e_{2}^{*}$ are proportional if and only if they belong to the same C1-set, if and only if, by Lemma 2.3.2 (iv), $\left(\left\{e_{1}, e_{2}\right\}\right)$ is separating pair of edges. Therefore the edges deleted to pass from $M^{*}(\Gamma)$ to $\widehat{M^{*}(\Gamma)}$ correspond exactly to the edges contracted to construct $\Gamma^{3}$ from $\Gamma$, and hence we get that $\widetilde{M^{*}(\Gamma)} \cong M^{*}\left(\Gamma^{3}\right)$.

5.2. The posets $\mathcal{O} \mathcal{P}_{\Gamma}$ and $\overline{\mathcal{O P}} \mathcal{P}_{\Gamma}$. We defined totally cyclic orientations in Definition 2.4.1. Now we introduce a partial ordering among them.

Definition 5.2.1. The poset $\mathcal{O} \mathcal{P}_{\Gamma}$ of totally cyclic orientations of $\Gamma$ is the set of pairs $\left(S, \phi_{S}\right)$ where $S \in \mathcal{S} \mathcal{P}_{\Gamma}$ and $\phi_{S}$ is a totally cyclic orientation of $\Gamma \backslash S$, endowed with the following partial order

$$
\left(S, \phi_{S}\right) \geq\left(T, \phi_{T}\right) \Leftrightarrow S \subset T \text { and } \phi_{T}=\left(\phi_{S}\right)_{\mid E(\Gamma \backslash T)} .
$$

We call $S$ the support of the orientation $\phi_{S}$.

We have a natural map

$$
\begin{gathered}
\text { Supp : } \mathcal{O P}_{\Gamma} \rightarrow \mathcal{S} \mathcal{P}_{\Gamma} \\
\left(S, \phi_{S}\right) \mapsto S
\end{gathered}
$$

which is order-preserving by definition and surjective because of Lemma 2.4.3(1).

We say that a map $\pi:(P, \leq) \rightarrow(Q, \leq)$ between two posets $(P, \leq)$ and $(Q, \leq)$ is a quotient if and only if for every $x, y \in Q$ we have that

$$
x \leq y \Leftrightarrow \text { there exist } \widetilde{x} \in \pi^{-1}(x) \text { and } \widetilde{y} \in \pi^{-1}(y) \text { such that } \widetilde{x} \leq \widetilde{y} .
$$

In particular $\pi$ is monotone and surjective. Observe also that if $\pi:(P, \leq) \rightarrow(Q, \leq)$ is a quotient, then $(P, \leq)$ is graded if and only if $(Q, \leq)$ is graded, and in this case we can choose two rank functions $\rho_{P}$ on $P$ and $\rho_{Q}$ on $Q$ such that $\rho_{Q}(\pi(x))=\rho_{P}(x)$.

We introduce now the outdegree function.

Definition 5.2.2. The outdegree function $\underline{d}^{+}$is the map

$$
\begin{aligned}
\underline{d}^{+}: \mathcal{O} \mathcal{P}_{\Gamma} & \longrightarrow \mathbb{N}^{V(\Gamma)} \\
\left(S, \phi_{S}\right) & \mapsto\left\{d^{+}\left(S, \phi_{S}\right)_{v}\right\}_{v \in V(\Gamma)},
\end{aligned}
$$

where $d^{+}\left(S, \phi_{S}\right)_{v}$ is the number of edges of $\Gamma \backslash S$ that are going out of the vertex $v$ according to the orientation $\phi_{S}$.

Note that $\underline{d}^{+}$is monotone with respect to the component-by-component partial order on $\mathbb{N}^{V(\bar{\Gamma})}$. Moreover

$$
\sum_{v \in V(\Gamma)} d^{+}\left(S, \phi_{S}\right)_{v}=\#(E(\Gamma \backslash S)) .
$$

This definition enables us to introduce an equivalence relation $\sim$ on $\mathcal{O} \mathcal{P}_{\Gamma}$. 
Definition 5.2.3. We say that two elements $\left(S, \phi_{S}\right)$ and $\left(S^{\prime}, \phi_{S^{\prime}}\right)$ of $\mathcal{O} \mathcal{P}_{\Gamma}$ are equivalent, and we write that $\left(S, \phi_{S}\right) \sim\left(S^{\prime}, \phi_{S^{\prime}}\right)$, if $S=S^{\prime}$ and $\underline{d}^{+}\left(S, \phi_{S}\right)=$ $\underline{d}^{+}\left(S^{\prime}, \phi_{S^{\prime}}\right)$. We denote by $\left[\left(S, \phi_{S}\right)\right]$ the equivalence class of $\left(S, \phi_{S}\right)$.

The set of equivalence classes will be denoted $\overline{\mathcal{O P}} \mathcal{P}_{\Gamma}:=\mathcal{O} \mathcal{P}_{\Gamma} / \sim$. On $\overline{\mathcal{O P}} \mathcal{P}_{\Gamma}$ we define a poset structure by saying that $\left[\left(S, \phi_{S}\right)\right] \geq\left[\left(T, \phi_{T}\right)\right]$ if there exist $\left(S^{\prime}, \phi_{S^{\prime}}\right) \sim$ $(S, \phi)$ and $\left(T^{\prime}, \phi_{T^{\prime}}\right) \sim\left(T, \phi_{T}\right)$ such that $\left(S^{\prime}, \phi_{S^{\prime}}\right) \geq\left(T^{\prime}, \phi_{T^{\prime}}\right)$ in $\mathcal{O} \mathcal{P}_{\Gamma}$.

Note that $\overline{\mathcal{O} \mathcal{P}_{\Gamma}}$ is a quotient of the poset $\mathcal{O} \mathcal{P}_{\Gamma}$ and that the natural map of posets Supp : $\mathcal{O P} \mathcal{P}_{\Gamma} \rightarrow \mathcal{S P}_{\Gamma}$ factors as

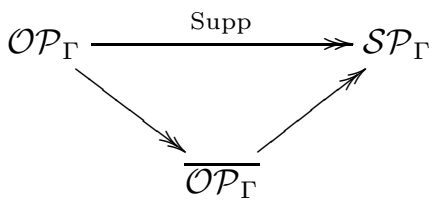

The next two lemmas show that $\mathcal{O} \mathcal{P}_{\Gamma}$ and $\overline{\mathcal{O} \mathcal{P}_{\Gamma}}$ are invariant under cyclic equivalence and 3-edge connectivization.

Lemma 5.2.4. The posets $\mathcal{O} \mathcal{P}_{\Gamma}$ and $\overline{\mathcal{O P}} \mathcal{P}_{\Gamma}$ depend only on $[\Gamma]_{\text {cyc }}$.

Proof. It is enough to show that the posets $\mathcal{O P}_{\Gamma}$ and $\overline{\mathcal{O} \mathcal{P}_{\Gamma}}$ do not change under the two moves of Theorem 2.2.4.

Consider first a move of type (1), that is the gluing of two graphs $\Gamma_{1}$ and $\Gamma_{2}$ at two vertices $v_{1} \in V\left(\Gamma_{1}\right)$ and $v_{2} \in V\left(\Gamma_{2}\right)$ (see figure 4). Call $\Gamma$ the resulting graph

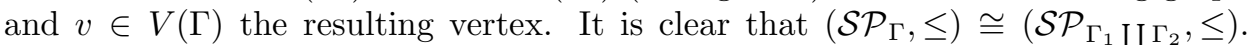
Given an element $S \in \mathcal{S} \mathcal{P}_{\Gamma}$, we denote by $\left(S_{1}, S_{2}\right)$ the corresponding element of $\mathcal{S} \mathcal{P}_{\Gamma_{1} \amalg \Gamma_{2}}$. It is easy to check that any totally cyclic orientation $\phi_{S}$ of $\Gamma$ $S$ induces totally cyclic orientations $\phi_{S_{1}}$ and $\phi_{S_{2}}$ of $\Gamma_{1} \backslash S_{1}$ and $\Gamma_{2} \backslash S_{2}$ and conversely. Moreover the outdegree $\underline{d}^{+}\left(S, \phi_{S}\right)$ determines, and is determined by, the two outdegrees $\underline{d}^{+}\left(S_{1}, \phi_{S_{1}}\right)$ and $\underline{d}^{+}\left(S_{2}, \phi_{S_{2}}\right)$, hence we get the desired conclusion.

Consider now a move of type (2). Let $\Gamma$ be obtained by gluing the two graphs $\Gamma_{1}$ and $\Gamma_{2}$ according to the rule $u_{1} \leftrightarrow u_{2}$ and $v_{1} \leftrightarrow v_{2}$, and let $\bar{\Gamma}$ be obtained by gluing $\Gamma_{1}$ and $\Gamma_{2}$ according to the rule $u_{1} \leftrightarrow v_{2}$ and $v_{1} \leftrightarrow u_{2}$ (see figure 3). Note that since $E(\Gamma)=E\left(\Gamma_{1}\right) \cup E\left(\Gamma_{2}\right)$, any element $S \in \mathcal{S} \mathcal{P}_{\Gamma}$ determines two subsets $S_{1} \subset E\left(\Gamma_{1}\right)$ and $S_{2} \in E\left(\Gamma_{2}\right)$. These two subsets $S_{1}$ and $S_{2}$ determine also a subset $\bar{S} \in E(\bar{\Gamma})$, which is easily seen to belong to $\mathcal{S P} \bar{\Gamma}$. The association $S \mapsto \bar{S}$ determines an isomorphism $\left(\mathcal{S} \mathcal{P}_{\Gamma}, \leq\right) \cong\left(\mathcal{S} \mathcal{P}_{\bar{\Gamma}}, \leq\right)$. We now construct, for any $S \in \mathcal{S P}_{\Gamma}$, a bijection between the set of all totally cyclic orientations (resp. totally cyclic orientations up to equivalence) on $\Gamma \backslash S$ and the set of totally cyclic orientations (resp. totally cyclic orientations up to equivalence) on $\Gamma \backslash \bar{S}$. Any orientation $\phi_{S}$ on $\Gamma \backslash S$ determines two orientations $\phi_{S_{1}}$ and $\phi_{S_{2}}$ on $\Gamma_{1} \backslash S_{1}$ and $\Gamma_{2} \backslash S_{2}$, respectively. We define an orientation $\phi_{\bar{S}}$ of $\bar{\Gamma} \backslash \bar{S}$ by putting together the orientation $\phi_{S_{1}}$ and the inverse of the orientation $\phi_{S_{2}}$, that is the orientation $\phi_{S_{2}}^{-1}$ obtained by reversing the direction of all the edges. Using Lemma 2.4.3, it is easy to check that if $\phi_{S}$ is a totally cyclic orientation of $\Gamma \backslash S$ then $\phi_{\bar{S}}$ is a totally cyclic orientation of $\bar{\Gamma} \backslash \bar{S}$. Moreover it is straightforward to check that the outdegree function $\underline{d}^{+}\left(S, \phi_{S}\right)$ determines and is completely determined by $\underline{d}^{+}\left(\bar{S}, \phi_{\bar{S}}\right)$. Clearly the association $\phi_{S} \mapsto \phi_{\bar{S}}$ is a bijection since we can reconstruct $\phi_{S}$ starting from $\phi_{\bar{S}}$ by reversing the orientation on $\Gamma_{2}$. Moreover it is easy to check that the constructed bijections $\mathcal{O} \mathcal{P}_{\Gamma} \cong \mathcal{O} \mathcal{P}_{\bar{\Gamma}}$ and $\overline{\mathcal{O P}} \mathcal{P}_{\Gamma} \cong \overline{\mathcal{O} \mathcal{P}_{\bar{\Gamma}}}$ are compatible with the poset structure, and thus we are done.

Lemma 5.2.5. For any choice of $\Gamma^{3}$ we have natural isomorphisms of posets: $\mathcal{O} \mathcal{P}_{\Gamma} \cong \mathcal{O} \mathcal{P}_{\Gamma^{3}}$ and $\overline{\mathcal{O P}} \mathcal{P}_{\Gamma} \cong \overline{\mathcal{O P} \Gamma_{\Gamma^{3}}}$. 
Proof. It is enough to show that the posets $\mathcal{O} \mathcal{P}_{\Gamma}$ and $\overline{\mathcal{O P}} \mathcal{P}_{\Gamma}$ do not change under the two moves of Definition 2.3.6.

Recall that for every $S \in \mathcal{S P}_{\Gamma}$ we have $E(\Gamma)_{\text {sep }} \subset S$. Therefore $E(\Gamma)_{\text {sep }}$ does not affect the totally cyclic orientations on $\Gamma \backslash S$, nor does it affect the outdegree function. This proves that $\mathcal{O P} \Gamma$ does not change when separating edges of $\Gamma$ get contracted.

Consider now a move of type (B), that is the contraction of an edge $e_{1}$ belonging to a separating pair $\left(e_{1}, e_{2}\right)$. We refer to the notations of figure 5 . We known that $\mathcal{S P}_{\Gamma} \cong \mathcal{S} \mathcal{P}_{\bar{\Gamma}}$, by Lemma 5.1 .10 . Given an element $S \in \mathcal{S P}_{\Gamma}$, we denote by $\bar{S}$ the corresponding element in $\mathcal{S} \mathcal{P}_{\bar{\Gamma}}$. We now construct, for any $S \in \mathcal{S P}_{\Gamma}$, a bijection between the set of all totally cyclic orientations (resp. totally cyclic orientations up to equivalence) on $\Gamma \backslash S$ and the set of totally cyclic orientations (resp. totally cyclic orientations up to equivalence) on $\Gamma \backslash \bar{S}$. If $\bar{e} \in \bar{S}$ (which happens exactly when $e_{1}, e_{2} \in S$ ), then $\bar{\Gamma} \backslash \bar{S}$ is cyclically equivalent to $\Gamma \backslash S$ and therefore we conclude by the previous Lemma. If $\bar{e} \notin \bar{S}$ (which happens exactly when $e_{1}$ and $e_{2}$ do not belong to $S$ ), we lift any totally cyclic orientation $\phi_{\bar{S}}$ of $\bar{\Gamma} \backslash \bar{S}$ to an orientation $\phi_{S}$ of $\Gamma \backslash S$ by orienting any edge in $E\left(\Gamma \backslash S \cup\left\{e_{1}\right\}\right)$ as the corresponding edge in $E(\bar{\Gamma} \backslash S)$, and by orienting $e_{1}$ so that the cycle $\Gamma\left(\left\{e_{1}, e_{2}\right\}\right)$ is cyclically oriented. Lemma 2.4 .3 implies that $\phi_{S}$ is a totally cyclic orientation of $\Gamma \backslash S$ and that any totally cyclic orientation $\phi_{S}$ must arise from a totally cyclic orientation of $\phi_{\bar{S}}$ via this construction. Moreover, it is easy to check that the outdegrees $d^{+}\left(S, \phi_{S}\right)$ and $d^{+}\left(\bar{S}, \phi_{\bar{S}}\right)$ are completely determined one from another, and this concludes the proof.

5.2.6. A conjectural geometric description of $\mathcal{O} \mathcal{P}_{\Gamma}$ and $\overline{\mathcal{O P}} \mathcal{P}_{\Gamma}$

We propose a conjectural geometric description of the two posets $\mathcal{O P} \mathcal{P}_{\Gamma}$ and $\overline{\mathcal{O P}} \bar{\Gamma}$. Recall the following definition (see for example [BdlHN97, Pag. 174]).

Definition 5.2.7. The Voronoi polyhedron of the graph $\Gamma$ is the compact convex polytope defined by

$$
\operatorname{Vor}_{\Gamma}:=\left\{x \in H_{1}(\Gamma, \mathbb{R}):(x, x) \leq(x-\lambda, x-\lambda) \text { for all } \lambda \in H_{1}(\Gamma, \mathbb{Z})\right\} .
$$

We denote with Faces $\left(\operatorname{Vor}_{\Gamma}\right)$ the poset of faces of the Voronoi polyhedron $\operatorname{Vor}_{\Gamma}$, with the order given by the reverse of the natural inclusion between the faces. It is a graded poset with minimum equal to the interior of $\operatorname{Vor}_{\Gamma}$ and normalized rank function equal to the codimension of the faces.

From the definition, it follows that $\operatorname{Vor}_{\Gamma}$ is a fundamental domain for the action of $H_{1}(\Gamma, \mathbb{Z})$ on $H_{1}(\Gamma, \mathbb{R})$ by translations. In particular $H_{1}(\Gamma, \mathbb{Z})$ acts by translation on the faces of $\operatorname{Vor}_{\Gamma}$. We denote with $\overline{\text { Faces }\left(\operatorname{Vor}_{\Gamma}\right)}$ the quotient poset of Faces $\left(\operatorname{Vor}_{\Gamma}\right)$ with respect to the action of $H_{1}(\Gamma, \mathbb{Z})$.

Conjecture 5.2.8. For a graph $\Gamma$, we have that

(i) $\mathcal{O} \mathcal{P}_{\Gamma} \cong \operatorname{Faces}\left(\operatorname{Vor}_{\Gamma}\right)$.

(ii) $\overline{\mathcal{O P}} \mathcal{P}_{\Gamma} \cong \overline{\operatorname{Faces}\left(\operatorname{Vor}_{\Gamma}\right)}$.

The above conjecture $(i)$ generalizes the bijection (proved in OS79, Prop. 5.2] and [BdlHN97, Prop. 6]) between the codimension-one faces of $\operatorname{Vor}_{\Gamma}$ and the oriented cycles of $\Gamma$ (which correspond to the elements $S \in \mathcal{O P}_{\Gamma}$ such that $b_{1}(\Gamma \backslash S)=$ 1). Therefore part $(i)$ proposes an answer to the interesting problem posed in [BdlHN97, Page 174]: "More ambitiously, one would like to understand the combinatorics of the Voronoi polyhedron in terms of oriented circuits of the graph".

\subsection{Conclusions.}

Lemma 5.3.1. The support map Supp : $\mathcal{O P}_{\Gamma} \longrightarrow \mathcal{S P}_{\Gamma}$ is a quotient of posets. Moreover, given $S, T \in \mathcal{S} \mathcal{P}_{\Gamma}$ such that $S$ covers $T$, and a totally cyclic orientation 
$\phi_{T}$ of $\Gamma \backslash T$, there are at most two (possibly equal) extensions of $\phi_{T}$ to a totally cyclic orientation $\phi_{S}$ of $\Gamma \backslash S$.

Proof. We already observed that Supp is surjective and order preserving. For the remaining part we use the fact that $\mathcal{S P}_{\Gamma}$ is graded by the function $b_{1}(\Gamma \backslash S)$ (see 5.1.8). By 5.1.10 and 5.2.5 we can assume that $\Gamma$ is 3 -edge connected. In particular, we have $E(\Gamma)_{\text {sep }}=\emptyset$.

It is easy to see that it suffices to assume $S=\emptyset$. The hypothesis that $\emptyset$ covers $T$ is equivalent to the fact that $b_{1}(\Gamma)=b_{1}(\Gamma \backslash T)+1$ or, equivalently, that $b_{1}(\Gamma(T))=1$. Hence $\Gamma(T)$ is a cycle (as $\left.E(\Gamma)_{\text {sep }}=\emptyset\right)$.

Using the characterization 2.4.3 (in particular part (2b) ) it is easy to check the only way to extend the orientation $\phi_{T}$ of $\Gamma \backslash T$ to a totally cyclic orientation on all of $\Gamma$ is by choosing for the edges of $T$ one of the two cyclic orientations of the cycle $\Gamma(T)$.

Summing up what we have proved in this section, we get the following

Theorem 5.3.2. Let $\Gamma$ and $\Gamma^{\prime}$ be two graphs. The following facts are equivalent:

(i) $\left[\Gamma^{3}\right]_{\mathrm{cyc}}=\left[\Gamma^{\prime 3}\right]_{\mathrm{cyc}}$.

(ii) $\operatorname{Del}(\Gamma) \cong \operatorname{Del}\left(\Gamma^{\prime}\right)$.

(iii) $\mathcal{S} \mathcal{P}_{\Gamma} \cong \mathcal{S} \mathcal{P}_{\Gamma^{\prime}}$ as posets.

(iv) $\mathcal{O} \mathcal{P}_{\Gamma} \cong \mathcal{O} \mathcal{P}_{\Gamma^{\prime}}$ as posets.

(v) $\overline{\mathcal{O P} \mathcal{P}_{\Gamma}} \cong \overline{\mathcal{O} \mathcal{P}_{\Gamma^{\prime}}}$ as posets.

Proof. The equivalence (ii) $\Leftrightarrow$ (iii) was proved in Proposition [3.2.3(iii), while the equivalence (ii) $\Leftrightarrow$ (iii) follows from Lemma 5.1.10. The implications (ii) $\Rightarrow$ (iv) and (ii) $\Rightarrow$ (v) follow from Lemmas 5.2 .4 and 5.2 .5 . Finally the implications (iv) $\Rightarrow$ (iii) and $(\mathbb{V}) \Rightarrow$ (iii) follows from the fact that $\mathcal{S} \mathcal{P}_{\Gamma}$ is a quotient poset of $\overline{\mathcal{O} \mathcal{P}_{\Gamma}}$ and $\mathcal{O P} \mathcal{P}_{\Gamma}$ (see Lemma 5.3.1 and the discussion after definition 5.2.3).

\section{Appendix A. Tropical Torelli map}

A.1. Tropical Geometry is a rather young area of mathematics, which has been developing fast in recent years. Nevertheless an exhaustive construction of the tropical analogues of certain fundamental algebro-geometric objects is not yet available in the literature; this is the case of the moduli spaces of curves and abelian varieties, in which we are here interested.

In this appendix, fixing $g \geq 2$ and assuming the existence and some properties of the moduli spaces $M_{g}^{\text {trop }}$ (of compact tropical curves of genus $g$ ) and $A_{g}^{\text {trop }}$ (of principally polarized tropical abelian varieties of dimension $g$ ), and of the tropical Torelli map $t_{g}^{\text {trop }}: M_{g}^{\text {trop }} \rightarrow A_{g}^{\text {trop }}$, we prove that the map $t_{g}^{\text {trop }}$ is of tropical degree one, as conjectured by Mikhalkin and Zharkov in [MZ07, Sect. 6.4]. This is done in Theorem A.2.1, whose proof relies on Theorem 4.1.9. The present appendix greatly benefited from several e-mail exchanges with G. Mikhalkin.

In this section we assume that all graphs are connected and all tropical curves are compact and connected. Recall that a graph is called 3-regular if all its vertices have valence 3 . We say that a tropical curve $C$ is 3 -regular if its associated graph is 3-regular (see Remark 4.1.3).

Remark A.1.1. A connected 3-regular graph of genus (i.e. first Betti number) $g$ has $3 g-3$ edges and $2 g-2$ vertices.

We will need the following easy

Lemma A.1.2. A 3-regular graph is 3-edge connected if and only if it is 3-connected. 
Proof. Since 3-connectivity always implies 3-edge-connectivity there is only one implication to prove. Let $\Gamma$ be a 3 -regular graph; suppose that $\Gamma$ is not 3-connected and let us prove that it is not 3-edge connected.

First of all, if $\Gamma$ has a loop based at a vertex $v$ then, as $v$ has valence 3 and the loop contributes by two to the valence of $v$, there is a unique other edge attached to $v$, which is necessarily a separating edge of $\Gamma$, so we are done. We can assume that $\Gamma$ has no loops.

Assume that $\Gamma$ has a separating vertex, $v$. Let $\Gamma^{\prime}$ be the graph obtained from $\Gamma$ by removing $v$ and all the edges adjacent to it. By assumption, $\Gamma^{\prime}$ is not connected. Since $v$ has valence 3 there is a connected component $\Gamma^{\prime \prime}$ of $\Gamma^{\prime}$ with the property that there exists a unique edge of $\Gamma$, call it $e$, connecting $v$ with one of the vertices of $\Gamma^{\prime \prime}$. Clearly $e$ is a separating edge of $\Gamma$, and we are done. We can assume that $\Gamma$ is 2-connected.

Since $\Gamma$ is not 3 -connected, there exists a separating pair of vertices, $\left\{v_{1}, v_{2}\right\}$, none of which is a separating vertex. Let $\Gamma^{\prime} \subset \Gamma$ be the graph obtained from $\Gamma$ by removing $v_{1}, v_{2}$, and all the edges adjacent to them. By assumption $\Gamma^{\prime}$ has at least two connected components. Since $v_{i}$ has valence 3 for $i=1,2$, there are at most two edges joining $v_{1}$ and $v_{2}$ (for otherwise $\Gamma$ has no other vertex and there is nothing to prove). Therefore there exists an edge, $e_{i}$, touching $v_{i}$ and such that the component of $\Gamma^{\prime}$ which touches $e_{i}$ touches no other edge adjacent to $v_{i}$. It is clear that the pair $\left\{e_{1}, e_{2}\right\}$ is a disconnecting pair of edges of $\Gamma$. Notice that the edge $e_{i}$ is not necessarily unique, but we are free to make a choice without changing the conclusion. The proof is complete.

We shall now make some reasonable assumptions on the moduli spaces $M_{g}^{\text {trop}}$, $A_{g}^{\text {trop }}$ and on the tropical map $t_{g}^{\text {trop }}$. After each set of assumptions we will provide motivations and, when possible, some references.

Assumption 1. There exists a moduli space $M_{g}^{\text {trop }}$ of dimension $3 g-3$ parametrizing tropical equivalence classes of (compact) tropical curves of genus $g . A$ generic point of $M_{g}^{\text {trop }}$ is a 3-regular tropical curve.

For a strategy to construct $M_{g}^{\text {trop }}$, see [Mik07a, Sect. 3.1].

A neighborhood of a 3-regular curve $C$ in $M_{g}^{\text {trop }}$ is obtained by varying the lengths $l(e)$ of the edges of the corresponding graph, and therefore, by Remark A.1.1, it is isomorphic to $\mathbb{R}_{>0}^{3 g-3} \subset \mathbb{R}^{3 g-3}$. This explains the dimension requirement.

The condition on the generic point (i.e. a point varying in an open dense subset of $M_{g}^{\text {trop }}$ ) derives from the fact that specializations of a tropical curve are obtained by letting some of its edge lengths go to 0 , i.e. by contracting some of its edges (see Mik07a, Sec.3.1.D]). Therefore, if we have a tropical curve $C_{0}$ with a vertex $v$ of valence $k+l \geq 4$, with $k, l \geq 2$, we can realize it as the limit of tropical curves $C_{t}$ in which the vertex $v$ is replaced by two vertices $v_{1}$ and $v_{2}$ of valence resp. $k+1$ and $l+1$ joined by a new edge $e$ :

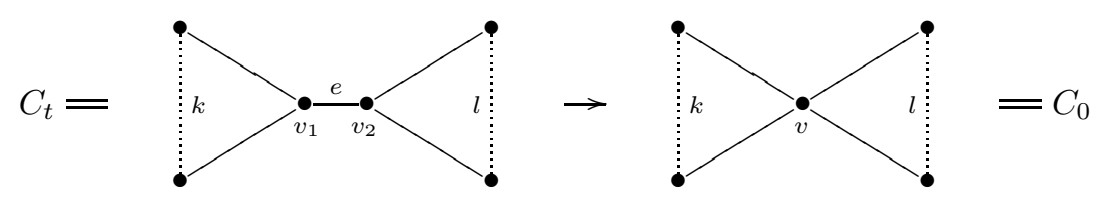

Figure $6 . C_{t}$ specializes to $C_{0}$ by letting $l(e) \rightarrow 0$. 
By applying the above procedure on all the vertices of valence greater than 3 , we obtain that every tropical curve is the limit of 3-regular tropical curves.

The previous discussion yields the following useful observation.

Remark A.1.3. Let $C_{0}$ be a tropical curve and $\left(\Gamma_{0}, l_{0}\right)$ the associated metric graph. To prove that $C_{0}$ is the specialization of tropical curves $C_{t}$ of a certain combinatorial type, i.e. having a certain associated graph $\Gamma_{t}$, is equivalent to proving that there exists a surjective edge-contracting map $\sigma: \Gamma_{t} \rightarrow \Gamma_{0}$, such that $b_{1}\left(\Gamma_{t}\right)=b_{1}\left(\Gamma_{0}\right)$.

Indeed, it suffices to define the length function $l_{t}$ for $C_{t}$ so that $l_{t}$ equals $l$ on the edges not contracted by $\sigma$, and $l_{t}$ tends to 0 on every edge contracted by $\sigma$.

Assumption 2. There exists a moduli space $A_{g}^{\text {trop }}$ of dimension $\left(\begin{array}{c}g+1 \\ 2\end{array}\right)$ parametrizing principally polarized tropical abelian varieties of dimension $g$.

Assumption 3. The Torelli map

$$
\begin{aligned}
t_{g}^{\text {trop }}: M_{g}^{\text {trop }} & \longrightarrow A_{g}^{\text {trop }} \\
C & \mapsto\left(\operatorname{Jac}(C), \Theta_{C}\right)
\end{aligned}
$$

is a tropical map. Its image will be denoted $J_{g}^{\text {trop }}:=t_{g}^{\text {trop }}\left(M_{g}^{\text {trop }}\right)$.

Locally around a 3-regular tropical curve $C, t_{g}^{\text {trop }}$ is given by the restriction of a linear map $L_{C}: \mathbb{R}^{3 g-3} \rightarrow \mathbb{R}^{\left(\begin{array}{c}g+1 \\ 2\end{array}\right)}$ which sends $\mathbb{Z}^{3 g-3}$ to $\mathbb{Z}^{\left(\begin{array}{c}g+1 \\ 2\end{array}\right) \text {. }}$

Note that $J_{g}^{\text {trop }}$ is a tropical variety. A neighborhood in $A_{g}^{\text {trop }}$ of $t_{g}^{\text {trop }}(C)$, for some $C \in M_{g}^{\text {trop }}$, is obtained by varying the entries of the symmetric $g \times g$ matrix associated to the scalar product $(,)_{l}$ on $H_{1}(C, \mathbb{R})$, and therefore it is isomorphic to

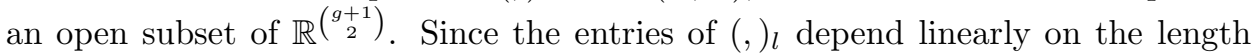
function $l$, we get that $t_{g}^{\text {trop }}$ is given locally around $C$ by the restriction of a linear

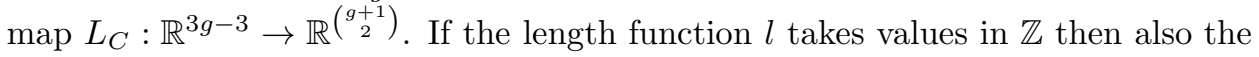
entries of $(,)_{l}$ with respect to a basis in $H_{1}(C, \mathbb{Z})$ will be in $\mathbb{Z}$.

Mikhalkin and Zharkov conjectured (in [MZ07, Sec. 6.4]) that $t_{g}^{\text {trop }}$, although not injective, is of tropical degree one to its image, in the following sense:

Definition A.1.4. The map $t_{g}^{\text {trop }}: M_{g}^{\text {trop }} \rightarrow J_{g}^{\text {trop }}$ is of tropical degree one if the following two conditions are satisfied:

(i) The inverse image via $t_{g}^{\text {trop }}$ of a generic point $t_{g}^{\text {trop }}(C)$ of $J_{g}^{\text {trop }}$ consists only of $C$.

(ii) For a generic point $t_{g}^{\text {trop }}(C)$ of $J_{g}^{\text {trop }}$, the linear map $L_{C}$ of Assumption 3 is primitive, i. e. $L_{C}^{-1}\left(\mathbb{Z}^{\left(\begin{array}{c}g+1 \\ 2\end{array}\right)}\right)=\mathbb{Z}^{3 g-3}$.

A.2. In this final part we shall prove the following

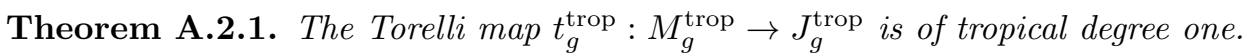

The proof will be at the end, combining Theorem 4.1.9 with Proposition A.2.4

A.2.2. Let $v \in V(\Gamma)$; we denote by $E_{v}(\Gamma)$ the set of edges of $\Gamma$ that are adjacent to the vertex $v$. Suppose that $v$ has valence $M \geq 4$. In the proof of the following proposition we will use an elementary operation, called a (valence reducing) extension of $\Gamma$ at $v$, which is a kind of inverse to the contraction of an edge in $v$, and which has the effect of replacing $v$ by two new vertices of valence at least 3 and strictly smaller than $M$. This will be a (not unique) graph $\Gamma^{\prime}$ endowed with a map $\Gamma^{\prime} \rightarrow \Gamma$ which is an isomorphism away from $v$ and which contracts a unique non loop edge, $e^{\prime}$, to $v$. Thus $\Gamma^{\prime}$ is obtained by replacing the vertex $v$ by an edge $e^{\prime}$ having extremal vertices $u^{\prime}$ and $w^{\prime}$, and by distributing between $u^{\prime}$ and $w^{\prime}$ the edges adjacent to $v$, so that both $u^{\prime}$ and $w^{\prime}$ have valence at least 3. More precisely, 
we denote $V(\Gamma)=\left\{v, v_{2} \ldots v_{\gamma}\right\}$ and $V\left(\Gamma^{\prime}\right)=\left\{u^{\prime}, w^{\prime}, v_{2}^{\prime} \ldots v_{\gamma}^{\prime}\right\}$ with $v_{i}$ corresponding to $v_{i}^{\prime}$ via the contraction map; similarly we denote $E(\Gamma)=\left\{e_{1} \ldots e_{\delta}\right\}$ and $E\left(\Gamma^{\prime}\right)=\left\{e^{\prime}, e_{1}^{\prime} \ldots e_{\delta}^{\prime}\right\}$. The edge $e^{\prime}$ joins $u^{\prime}$ to $w^{\prime}$; if an edge $e_{i}$ joins $v_{j}$ to $v_{i}$, then $e_{i}^{\prime}$ joins $v_{j}^{\prime}$ to $v_{i}^{\prime}$. If an edge $e_{i}$ joins $v$ to $v_{i}$ we need to specify whether $e_{i}^{\prime}$ joins $u^{\prime}$ to $v_{i}^{\prime}$, or $w^{\prime}$ to $v_{i}^{\prime}$ (which is why $\Gamma^{\prime}$ is not unique). For whichever choice we make, provided that $u^{\prime}$ and $w^{\prime}$ have valence at least 3 (this is possible as $M \geq 4$, and there is $e^{\prime}$ joining $u^{\prime}$ with $\left.w^{\prime}\right)$ it is clear that there is a map $\Gamma^{\prime} \rightarrow \Gamma$ as wanted.

Remark A.2.3. Let $\Gamma$ be 3-edge conected, and let $\Gamma^{\prime} \rightarrow \Gamma$ be an extension of $\Gamma$ at a vertex $v$, such that $e^{\prime}$ is not a separating edge of $\Gamma^{\prime}$. Then any separating pair of edges of $\Gamma^{\prime}$ is of type $\left(e^{\prime}, e_{i}^{\prime}\right)$ with $e_{i}$ a separating edge of $\Gamma \backslash v$.

Here and in the sequel $\Gamma \backslash v=\Gamma \backslash\{v\}$ (we omit the braces to ease the notation) denotes the topological space obtained by removing the point corresponding to the vertex $v$ from the topological space naturally associated to $\Gamma$. In particular, $\Gamma \backslash v$ is not a graph, but we will extend to it the terminology used for graphs, as no confusion is likely to arise.

Proposition A.2.4. The locus in $M_{g}^{\text {trop }}$ of 3-edge connected curves is equal to the closure of the locus of 3-regular, 3-connected curves.

Proof. Recall that, by Lemma A.1.2 a 3-regular, 3-edge connected graph is also 3-connected. Therefore, by remark A.1.3 it is enough to show the following. There exists a 3-regular, 3-edge connected graph $\Gamma^{*}$ together with a surjective edge-contracting map $\Gamma^{*} \rightarrow \Gamma$ such that $b_{1}(\Gamma)=b_{1}\left(\Gamma^{*}\right)$ if and only if $\Gamma$ is 3-edge connected.

The fact that if $\Gamma^{*}$ is 3-regular and 3-edge connected then $\Gamma$ is 3-edge connected follows from the easy fact that the edge connectivity cannot decrease by edge contraction.

Conversely, consider a 3 -edge connected graph $\Gamma$; note that $\Gamma$ has valence at least 3. Let $M$ be the maximum valence of a vertex of $\Gamma$; if $M=3$ there is nothing to prove, so suppose $M \geq 4$. We shall prove that a $\Gamma^{*}$ as above exists by constructing a 3-edge connected graph $\Gamma^{\prime}$ which is an extension of $\Gamma$, and such that the number of $M$-valent vertices of $\Gamma^{\prime}$ is less than that of $\Gamma$. It is clear that this will be enough. Let $v$ be a vertex of valence $M$.

Step 1. Suppose that $v$ is the base of some loop $e_{\ell}$ of $\Gamma$. Call $G$ the complement in $\Gamma$ of $v$ and of all loops based at $v$

$$
G:=\Gamma \backslash v \backslash \bigcup_{e_{\ell} \text { loop based at } v} e_{\ell} .
$$

If $G$ is not empty there are at least 3 edges $e_{1}, e_{2}, e_{3}$ in $E_{v}(\Gamma)$ contained in $G$ (as $\Gamma$ is 3 -edge connected,). We apply an extension of $\Gamma$ at $v$ so that for every loop $e_{\ell}$ the corresponding $e_{\ell}^{\prime}$ joins $u^{\prime}$ with $w^{\prime}$ (notation in A.2.2), $e_{1}^{\prime}$ is adjacent to $u^{\prime}$ and $e_{2}^{\prime}, e_{3}^{\prime}$ are adjacent to $w^{\prime}$. If there are other edges $e_{4}, \ldots, e_{m} \in E_{v}(\Gamma)$, we distribute $e_{4}^{\prime}, \ldots, e_{m}^{\prime}$ between $E_{u^{\prime}}\left(\Gamma^{\prime}\right)$ and $E_{w^{\prime}}\left(\Gamma^{\prime}\right)$ arbitrarily. It is clear that $u^{\prime}$ and $w^{\prime}$ have valence at most $M-1$. Now notice that both $e^{\prime}$ and $e_{\ell}^{\prime}$ join $u^{\prime}$ with $w^{\prime}$. Therefore $e^{\prime}$ is not a separating edge of $\Gamma^{\prime}$, and there exists no separating pair of edges $\left(e^{\prime}, e_{i}^{\prime}\right)$ unless $e_{i}^{\prime}=e_{\ell}^{\prime}$ for some loop $e_{\ell}$ based at $v$. It is obvious that $e_{\ell}$ is not a separating edge of $\Gamma \backslash v$; by Remark A.2.3 we have that $\Gamma^{\prime}$ is 3-edge connected, so we are done.

Repeating Step 1 we reduce to the case when $v$ is not the base of any loop.

Step 2. Consider $\Gamma \backslash v$ and suppose it is not connected, i.e. $v$ is a separating vertex of $\Gamma$. Then $\Gamma \backslash v$ contains no separating edges. Call $G_{1}, \ldots G_{c}$ the connected components of $\Gamma \backslash v$. Since $\Gamma$ is 3 -edge connected every $G_{i}$ contains at least 3 edges adjacent to $v$; call them $e_{1}^{i}, e_{2}^{i}, e_{3}^{i}$. Now apply an extension of $\Gamma$ at $v$ so that for every odd $i \leq c$ (respectively for every even $i \leq c$ ) $e_{1}^{i}$ is adjacent to $u^{\prime}$ (resp. to 
$w^{\prime}$ ) and $e_{2}^{i}, e_{3}^{i}$ are adjacent to $w^{\prime}$ (resp. to $u^{\prime}$ ). In this way $e^{\prime}$ is not a separating edge of $\Gamma^{\prime}$, and hence $\Gamma^{\prime}$ is 3-edge connected (by Remark A.2.3). By distributing the remaining (if any) edges coming from $E_{v}(\Gamma)$ between $w^{\prime}$ and $u^{\prime}$ we have that, since $c \geq 2, u^{\prime}$ and $w^{\prime}$ have smaller valence than $v$. So we are done.

Step 3. Now suppose $\Gamma \backslash v$ connected and free from separating edges. Let $\Gamma^{\prime}$ be a valence reducing extension of $\Gamma$ at $v$ such that both $u^{\prime}$ and $w^{\prime}$ are at least 3 -valent. Now $e^{\prime}$ is not a separating edge of $\Gamma^{\prime}(v$ is not a separting vertex of $\Gamma)$ and hence by Remark A.2.3, $\Gamma^{\prime}$ ie 3-edge connected, as wanted..

Step 4. Finally, suppose $\Gamma \backslash v$ connected and admitting a non empty set, $S_{v} \subset E(\Gamma)$, of separating edges. An example of the construction we are going to illustrate is in Figure 7. Note that no edge in $S_{v}$ is adjacent to $v$, for otherwise $\Gamma \backslash v$ would be disconnected. Also, for any extension of $\Gamma$ at $v$, the new edge $e^{\prime}$ is not separating.

Consider the graph $\widetilde{\Gamma}:=\Gamma \backslash S_{v}$. The valence of $v$ as a vertex of $\widetilde{\Gamma}$ is again $M \geq 4$. If $\widetilde{\Gamma}$ fails to be connected, we replace it by the connected component containing $v$, which will not affect the conclusions.

It is clear that describing an extension $\Gamma^{\prime}$ of $\Gamma$ at $v$ is equivalent to describing an extension $\widetilde{\Gamma}^{\prime}$ of $\widetilde{\Gamma}$ at $v$ (one just needs to glue back to $\widetilde{\Gamma}^{\prime}$ the edges in $S_{v}$ and the connected components of $\Gamma \backslash S_{v}$ not containing $v$ ). Now, for $\Gamma^{\prime}$ to be 3 -edge connected we need to extend $\widetilde{\Gamma}$ so that, for any $e_{s} \in S_{v}$, the pair $\left(e^{\prime}, e_{s}^{\prime}\right)$ is not a separating pair of $\Gamma^{\prime}$, i.e. (since $e^{\prime}$ is not a separating edge of $\Gamma^{\prime}$ ) so that $e^{\prime}$ is not a separating edge of $\Gamma^{\prime} \backslash e_{s}^{\prime}$.

The space $\widetilde{\Gamma} \backslash v$ is not connected and we proceed like for Step 2, notice however that $\widetilde{\Gamma}$ is only 2 -edge connected. Call $H_{1}, \ldots H_{h}$ the connected components of $\widetilde{\Gamma} \backslash v$. As $\widetilde{\Gamma}$ is 2-edge connected each $H_{i}$ contains at least 2 edges adjacent to $v$; call them $e_{1}^{i}, e_{2}^{i}$. Now apply an extension of $\widetilde{\Gamma}$ at $v$ so that for every odd $i$ (resp. for every even $i$ ) $e_{1}^{i}$ is adjacent to $u^{\prime}$ (resp. to $w^{\prime}$ ) and $e_{2}^{i}$ is adjacent to $w^{\prime}$ (resp. to $u^{\prime}$ ). By doing this, $e^{\prime}$ is not a separating edge of $\widetilde{\Gamma}^{\prime}=\Gamma^{\prime} \backslash S_{v}$, and hence a fortiori $e^{\prime}$ is not a separating edge of $\Gamma^{\prime} \backslash e_{s}^{\prime}$. As we observed above, this implies that $\Gamma^{\prime}$ is 3-edge connected. By distributing between $w^{\prime}$ and $u^{\prime}$ the remaining edges we have that, as $h \geq 2$, the vertices $u^{\prime}$ and $w^{\prime}$ have smaller valence than $v$.

Since the removal of $S_{v}$, or of components of $\Gamma \backslash S_{v}$ not containing $v$, does not alter $\Gamma$ at $v$, we are done.

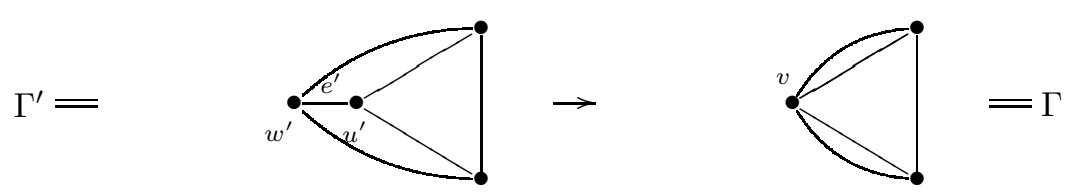

FiguRE 7. 3-edge connected extension of $\Gamma$ at $v$.

Proof of Theorem A.2.1. We claim that the generic point of $J_{g}^{\text {trop }}$ is of the form $t_{g}^{\text {trop }}(C)$ for a 3-regular and 3-connected curve $C$. It is clear that the generic point of $J_{g}^{\text {trop }}$ is the image of some generic point of $M_{g}^{\text {trop }}$, i.e. of some 3-regular curve. Let $C$ be a 3-regular curve not necessarily 3-connected. By Theorem 4.1.9 we have $t_{g}^{\text {trop }}(C)=t_{g}^{\text {trop }}\left(C^{3}\right)$, where $C^{3}$ is the 3 -edge connected curve associated to a 3-edge connectivization (see 4.1.7) of the metric graph of $C$. By Proposition A.2.4, $C^{3}$ is the specialization of a 3-regular, 3-connected curve. Therefore $t_{g}^{\operatorname{trop}}\left(C^{3}\right)=t_{g}^{\text {trop }}(C)$ is the specialization of the Torelli image of a 3-regular 3-connected curve. This proves the claim. 
Now, for a 3-connected curve $C$, Theorem4.1.9 implies that $\left(t_{g}^{\text {trop }}\right)^{-1}\left(t_{g}^{\text {trop }}(C)\right)=$ $\{C\}$ and therefore condition (ii) of Definition A.1.4 is satisfied.

Consider now the linear map $L_{C}$ of Assumption 3 . By what we just proved we can take $C$ a 3 -connected, 3-regular curve. To prove that $L_{C}$ is primitive, we have to show that if the scalar product $(,)_{l}$ on $H_{1}(C, \mathbb{R})$ takes integer values on $H_{1}(C, \mathbb{Z})$ then the length function $l$ takes also integer values.

Recall that, by Corollary 2.3.4, to say that $C$ is 3 -edge connected is to say that for every edge $e$ of the graph $\Gamma$ associated to $C$ the set $\{e\}$ is a C1-set of $\Gamma$. Hence, using Lemma 3.3.1, we deduce that for every $e$ there exist two cycles, $\Delta_{1}$ and $\Delta_{2}$, of $\Gamma$ such that $\{e\}=E\left(\Delta_{1}\right) \cap E\left(\Delta_{2}\right)$. Therefore there exist two elements $c_{1}, c_{2} \in H_{1}(C, \mathbb{Z})$ such that $l(e)=\left(c_{1}, c_{2}\right)_{l}$. Since by assumption $(,)_{l}$ takes integer values on $H_{1}(C, \mathbb{Z})$ we get that $l(e)=\left(c_{1}, c_{2}\right)_{l} \in \mathbb{Z}$ for every edge $e$ of $\Gamma$.

The proof of Theorem A.2.1 is complete.

\section{REFERENCES}

[Ale04] Alexeev, V.: Compactified Jacobians and Torelli map. Publ. RIMS, Kyoto Univ. 40 (2004), 1241-1265.

[Art06] Artamkin, I. V.: The discrete Torelli theorem. (Russian) Mat. Sb. 197 (2006), 3-16. English translation in Sb. Math. 197 (2006), 1109-1120.

[BdlHN97] Bacher, R., de la Harpe, P., Nagnibeda, T.: The lattice of integral flows and the lattice of integral cuts on a finite graph. Bull. Soc. Math. France 125 (1997), 167-198.

[BN07] Baker, M., Norine, S.: Riemann-Roch and Abel-Jacobi theory on a finite graph. Adv. Math. 215 (2007), 766-788.

[Big97] Biggs, N. L.: Algebraic potential theory on graphs. Bull. London Math. Soc. 29 (1997), 641-682.

[Cap08] Caporaso, L.: Geometry of the theta divisor of a compactified Jacobian. To appear in Journ. of the European Math. Soc. (available at arXiv:0707.4602).

[CV] Caporaso, L., Viviani, F.: Torelli theorem for stable curves. Preprint available at arXiv:0904.4039

[Die97] Diestel, R.: Graph theory. Graduate Text in Math. 173, Springer-Verlag, Berlin, 1997.

[Gat06] Gathmann, A.: Tropical algebraic geometry. Jahresber. Dtsch. Math.-Ver. 108, No. 1, 3-32 (2006).

[KS00] Kotani, M., Sunada, T.: Jacobian tori associated with a finite graph and its abelian covering graphs. Adv. in Applied Math. 24 (2000), 89-110.

[Mik05] Mikhalkin, G.: Enumerative tropical algebraic geometry in $\mathbb{R}^{2}$. J. Am. Math. Soc. 18 (2005), 313-377.

[Mik06] Mikhalkin, G.: Tropical geometry and its applications. International Congress of Mathematicians. Vol. II, 827-852, Eur. Math. Soc., Zürich, 2006.

[Mik07a] Mikhalkin, G.: Introduction to Tropical Geometry (notes from the IMPA lectures in Summer 2007) arXiv:0709.1049.

[Mik07b] Mikhalkin, G.: What is... a tropical curve? Notices Amer. Math. Soc. 54 (2007), 511-513.

[MZ07] Mikhalkin, G., Zharkov, I.: Tropical curves, their Jacobians and Theta functions. Contemporary Mathematics 465: Proceedings of the International Conference on Curves and Abelian Varieties in honor of Roy Smith's 65th birthday. 203-231.

[Nam76] Namikawa, Y: A new compactification of the Siegel Space and Degeneration of Abelian Varieties II. Math. Ann. 221, (1976) 201-241.

[OS79] Oda, T., Seshadri, C. S.: Compactifications of the Generalized Jacobian Variety. Trans. American Math. Soc. 253 (1979), 1-90.

[Oxl92] Oxley, J. G.: Matroid theory. Oxford Graduate Texts in Mathematics no. 3, Oxford Science Publications, 1992.

[RGST05] Richter-Gebert, J., Sturmfels, B., Theobald, T.: First steps in tropical geometry. In Proc. Conference on Idempotent mathematics and mathematical physics, Vienna 2003 (G.L. Litvinov and V.P. Maslov, eds.). Providence, RI: American Mathematical Society (AMS). Contemporary Mathematics 377, 289-317 (2005).

[Rob39] Robbins H. E.: A theorem on graphs with an application to a problem of trafic control. The American Mathematical Monthly 46 (1939), 281-283.

[Whi33] Whitney, H.: 2-isomorphic graphs. Amer. Journ. Math. 55 (1933), 245-254. 
Dipartimento di Matematica, Università Roma Tre, Largo S. Leonardo Murialdo 1, 00146 Roma (ItAly)

E-mail address: caporaso@mat.uniroma3.it

Dipartimento di Matematica, Università Roma Tre, Largo S. Leonardo Murialdo 1, 00146 Roma (Italy)

E-mail address: viviani@mat.uniroma3.it 